\title{
Featherweight Jigsaw A minimal core calculus for modular composition of classes ${ }^{\star}$
}

\author{
Giovanni Lagorio, Marco Servetto, and Elena Zucca \\ DISI, Univ. of Genova, v. Dodecaneso 35, 16146 Genova, Italy \\ email: \{lagorio, servetto, zucca\}@disi.unige.it
}

\begin{abstract}
We present FJIG, a simple calculus where basic building blocks are classes in the style of Featherweight Java, declaring fields, methods and one constructor. However, inheritance has been generalized to the much more flexible notion originally proposed in Bracha's Jigsaw framework. That is, classes play also the role of modules, that can be composed by a rich set of operators, all of which can be expressed by a minimal core.

We keep the nominal approach of Java-like languages, that is, types are class names. However, a class is not necessarily a structural subtype of any class used in its defining expression.

The calculus allows the encoding of a large variety of different mechanisms for software composition in class-based languages, including standard inheritance, mixin classes, traits and hiding. Hence, FJig can be used as a unifying framework for analyzing existing mechanisms and proposing new extensions.

We provide two different semantics of an FJIG program: flattening and direct semantics. The difference is analogous to that between two intuitive models to understand inheritance: the former where inherited methods are copied into heir classes, and the latter where member lookup is performed by ascending the inheritance chain. Here we address equivalence of these two views for a more sophisticated composition mechanism.
\end{abstract}

\section{Introduction}

Jigsaw is a framework for modular composition largely independent of the underlying language, designed by Gilad Bracha in his seminal thesis [7], and then formalized by a minimal set of operators in module calculi such as [19,2]. In this paper, we define an instantiation of Jigsaw, called Featherweight Jigsaw (FJIG for short), where basic building blocks are classes in the style of Java-like languages. That is, classes are collections of fields, methods and constructors, that can be instantiated to create objects; also, class names are used as types (nominal typing).

* This work has been partially supported by MIUR EOS DUE - Extensible Object Systems for Dynamic and Unpredictable Environments. 
The motivation for this work is that, even though Jigsaw has been proposed a long time ago and since then it has been greatly influential ${ }^{1}$, its design has been never fully exploited in the context of Java-like languages, as recently pointed out as an open question in [3]. Here, we provide a foundational answer to this question, by defining a core language which, however, embodies the key features of Java-like languages, in the same spirit of Featherweight Java [14] (FJ for short). Indeed, formally, a basic class of FJIG looks very much as a class in FJ. However, standard inheritance has been replaced by the much more flexible (module) composition, that is, by the rich set of operators of the Jigsaw framework.

Instantiating Jigsaw on Java-like languages poses some non trivial design problems. Just to mention one (others are discussed in Section 1), we keep the nominal approach of Java-like languages, that is, types are class names, however, a class is not necessarily a structural subtype of any class used in its defining expression. While this allows a more flexible reuse, it may prevent the (generalized) inheritance relation to be a subtyping relation. So, the required subtyping relations among classes are declared by the programmer and checked by the type system. Another challenging issue is the generalization to F JIG of two intuitive models to understand inheritance: one where inherited methods are copied into heir classes, and the other one where member lookup is performed by ascending the inheritance chain. We address the equivalence of these two views for a much more sophisticated composition mechanism. Formally, we provide two different semantics for an FJIG program: flattening semantics, that is, by translation into a program where all composition operators have been performed, and direct semantics, that is, by formalizing a dynamic look-up procedure.

The paper is organized as follows. Section 1 provides an informal introduction to F.JIG by using a sugared surface syntax. Section 2 introduces a lower level syntax and defines flattening semantics. Section 3 defines the type system and states its soundness. Section 4 defines direct semantics of FJIG and states the equivalence between the two semantics. In the Conclusion, we summarize the contribution of the paper and briefly discuss related and further work.

A preliminary version of this paper, focused on the equivalence between flattening and direct semantics, and not including the type system, is [15].

\section{An informal introduction}

In this section we illustrate the main features of FJIG by using a sugared surface syntax, given in Figure 1. We assume infinite sets of class names $C$, (member) names $N$, and variables $x$. We use the bar notation for sequences, e.g., $\bar{\mu}$ is a metavariable for sequences $\mu_{1} \ldots \mu_{n}$.

\footnotetext{
${ }^{1}$ Just to mention two different research areas, Jigsaw principles are present in work on extending the ML module system with mutually recursive modules [8,12,13], and Jigsaw operators already included those later used in mixin classes and traits $[10,1,18,9,17]$.
} 


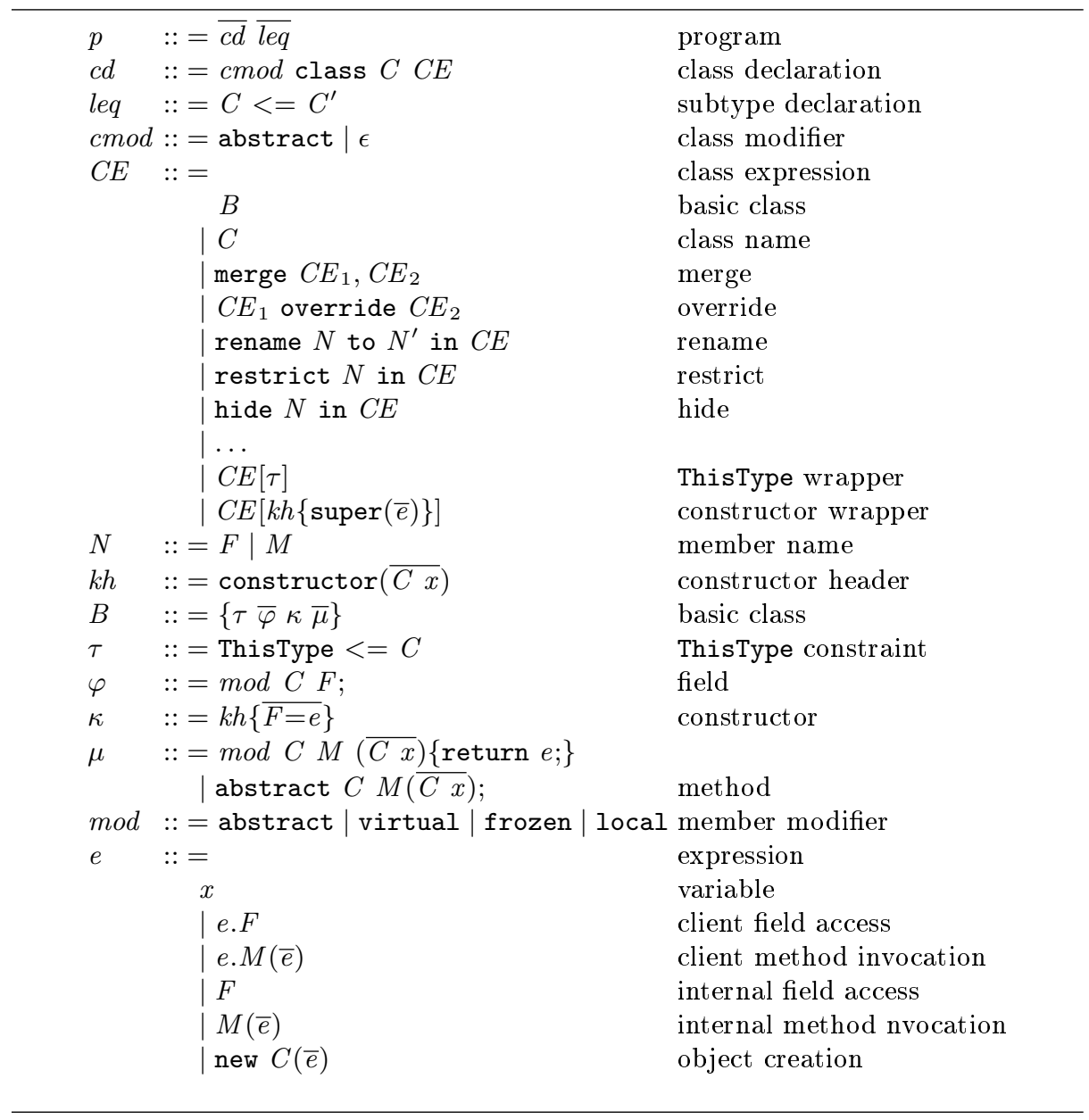

Fig. 1. FJIG (surface) syntax

This syntax is designed to keep a Java-like flavour as much as possible. In the next section we will use a lower-level representation, which allows to formalize the semantics in a simpler and natural way.

We will first revise Jigsaw features in the context of FJiG, then discuss some issues that are specific to the instantiation on Java-like languages.

Basic classes Jigsaw is a programming paradigm based on (module) composition, where a basic module (in our case, a class) is a collection of components (in our case, members), which can be of four different kinds, indicated by a modifier: abstract, virtual, frozen, and local. A method has no body if and only if its modifier is abstract. The meaning of modifiers is as follows: 
- An abstract member has no definition, and is expected to be defined later when composing the class with others.

- A virtual or frozen member has a definition, which can be changed by using the composition operators. However, the redefinition of a frozen member does not affect the other members, which still refer to its original definition.

- Finally, as the name suggests, a local member cannot be selected by a client, and is not affected by composition operators, hence its definition cannot be changed.

We assume by default (hence omit) the modifier frozen for fields and virtual for methods. A class having at least one abstract member must be declared abstract.

The following example shows two basic classes. ${ }^{2}$

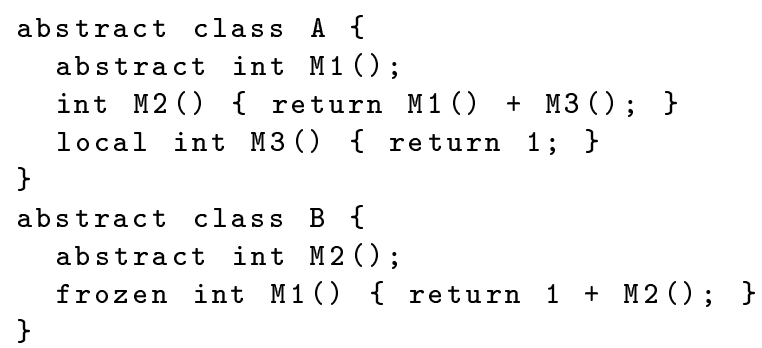

These two classes are abstract (hence cannot be instantiated).

Merge and override operators A concrete class can be obtained by applying the merge operator as follows:

class C merge A, B

This declaration is equivalent to the following:

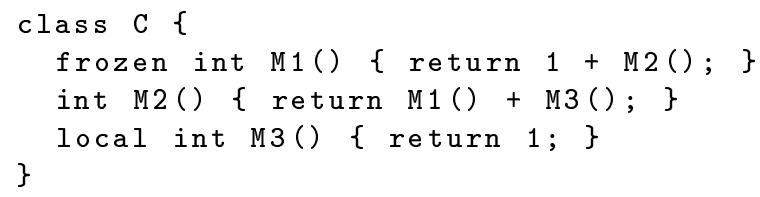

Conflicting definitions for the same (non-local) member are not permitted, whereas abstract members with the same name are shared. Members can be selected by client code unless they are local, that is, we can write, e.g., new C().M2() but not new $\mathrm{C}() . \mathrm{M} 3()$. To show the difference between virtual and frozen members, in the next examples we use the override operator, a variant of merge where conflicts are allowed and the left argument has the precedence.

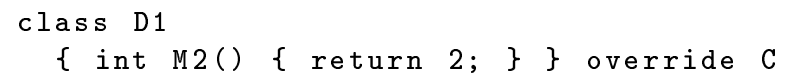

An invocation new D1().M2() will evaluate to 2, and an invocation new D1().M1() to 3 . On the other hand, in this case:

\footnotetext{
${ }^{2}$ To write more readable examples, we assume that the primitive type int and its operations are available.
} 
class D2

$\{$ int M1() \{return $3 ;\}$ \} override C

an invocation new D2().M1 () will evaluate to 3, but an invocation new D2().M2() will not terminate, since the internal invocation M1() in the body of M2() still refers to the old definition.

Client and internal member selection In a programming paradigm based on module composition, a module component can be either selected by a client, or used by other components inside the module itself. Correspondingly, in FJIG we distinguish between client field accesses and method invocations, which specify a receiver, and internal field accesses and method invocations, whose implicit receiver is the current object. Note that e.M(...) behaves differently from $M(.$. even in the case $e$ denotes an object of the same class (that is, internal selection does not correspond to selection of private members as in, e.g., Java). For instance, consider the following class, where we use the operator rename, which changes the name of a member.

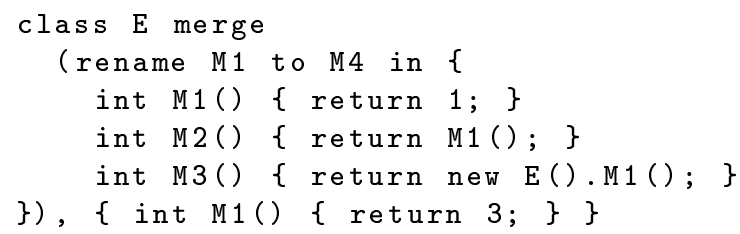

An invocation new E() . M2() returns 1, since the internal invocation in the body of M2 refers to the method now called M4. However, an invocation new E().M3() returns 3 , since the client invocation in the body of M3 refers to method M1 in E. Note that this does not even coincide with privateness on a "per object" basis as, e.g., in Smalltalk, since this would be the case even with a client invocation e.M1(), where $e$ denotes, as special case, the current object.

Other operators of the Jigsaw framework, besides the ones mentioned above, are restrict, which eliminates the definition for a member ${ }^{3}$, and hide, which makes a member no longer accessible from the outside. We refer to [7] and [2] for more details. All these operators and many others can be easily encoded (see [2]) by using a minimal set of primitive operators: sum, reduct, and freeze, which will be formally defined in next section.

We discuss now the issues specific to the instantiation on Java-like classes.

Fields and constructors It turns out that the above modifiers can be smoothly applied to fields as well, with analogous meaning, as shown by the following example which also illustrates how constructors work.

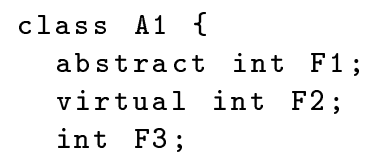

${ }^{3}$ Indeed, override can be obtained by combining merge and restrict. 


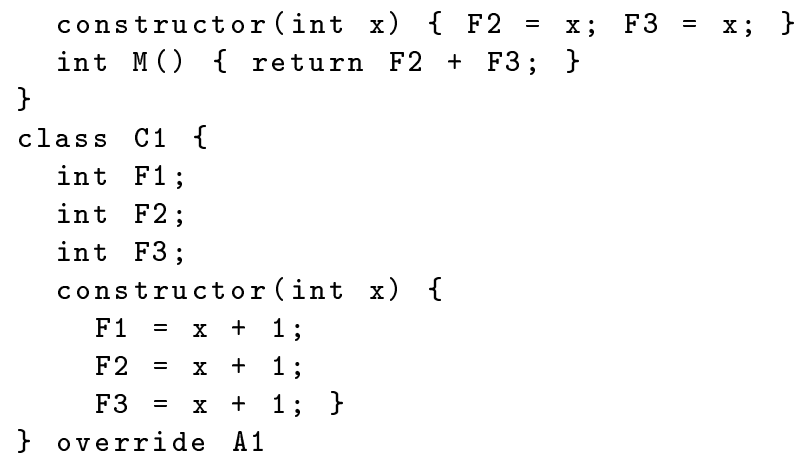

A basic class defines one ${ }^{4}$ constructor which specifies a sequence of parameters and a sequence of initialization expressions, one for each non-abstract field. We assume a default constructor with no parameters for classes having no fields. Note the difference with FJ, where the class constructor has a canonical form (parameters exactly correspond to fields). This would be inadequate in our framework since object layout must be hidden to clients. In order to be composed by merge/overriding, two classes should provide a constructor with the same parameter list (if it is not the case, a constructor wrapper can be inserted, see the last example of this section), and the effect is that the resulting class provides a constructor with the same parameter list, that executes both the original constructors. An instance of class $\mathrm{C} 1$ has five fields, and an invocation new C1(5).M() will return 11, since F2 in the body of $M$ refers to the field declared in $\mathrm{C} 1$ (initialized with 5+1), while F3 refers to the field declared in A1 (initialized with 5). Classes composed by merge/overriding can share the same field, provided it is abstract in all except (at most) one. Note that this corresponds to sharing fields as in, e.g., [4]; however, in our framework we do not need an ad-hoc notion.

Inheritance and subtyping Since our aim is to instantiate the Jigsaw framework on a Java-like language, we keep a nominal approach, that is, types are class names. However, subtyping does not coincide with the generalized inheritance relation, since some of the composition operators (e.g., renaming) do not preserve structural subtyping. Hence, we assume that a program includes a sequence of subtyping relations among classes explicitly declared by the programmer, and the type system checks, for each $C<=C^{\prime}$ subtype declaration, that the relation can be safely assumed since $C$ is a structural subtype of $C^{\prime} .^{5}$

Type of the current object The following code

\{

C M() $\{$ return this; $\}$

\footnotetext{
${ }^{4}$ Since overloading is not allowed.

${ }^{5}$ Alternatively, the compiler could (easily, since class types must be computed in any case) check which declared classes are structural subtype of each other and provide this information to the programmer. The former solution gives more control to the programmer at the price of more work.
} 
can be safely inherited only by classes which are a subtype of C. To ensure this, basic classes can declare a ThisType constraint:

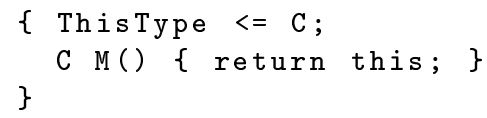

This constraint is used to typecheck the occurrences of this inside method bodies. Moreover, the constraint is checked when inheriting the code:

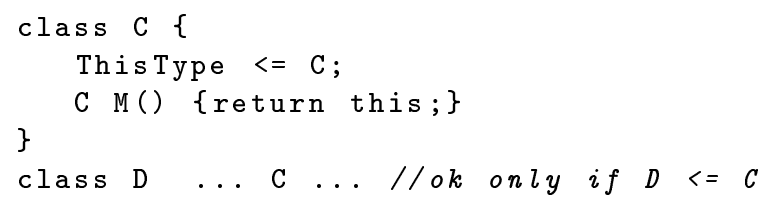

The ThisType constraint can be strengthened by the ThisType wrapping operator

C [ThisType $<=\mathrm{D}] / / O k$ only if $D<=C$

We assume a default constraint ThisType $<=$ Object, where Object is a predefined class with no members.

To conclude this section, we show a more significant example, where we also assume to have the type void and some statements in the syntax.

The following class DBSerializer, an example of the pattern template method [11], contains the method execute that opens a connection to a database and writes some data. While the behaviour of execute is fixed, the details on how to open the connection are left unspecified, and the implementation of the method serialize can be changed. This is reflected by the method modifiers. Class DBConnection is a given library class.

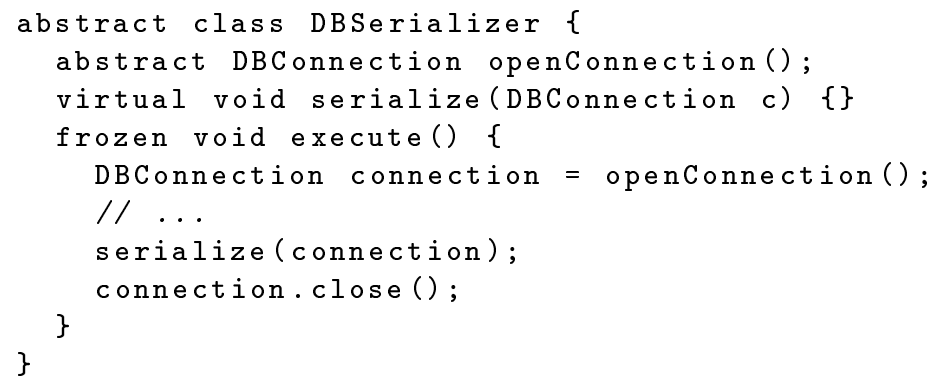

Suppose we want to specialize the class DBSerializer for the DB server MySQL. We can create this specialization, called MySQLSerializer, in two steps: first, we provide an implementation of method openConnection with the specific code for MySQL, then we hide it, since clients of MySQLSerializer should never invoke this method directly.

We start by defining an auxiliary class_MySQLSerializer, merging DBSerializer with an anonymous basic class: 


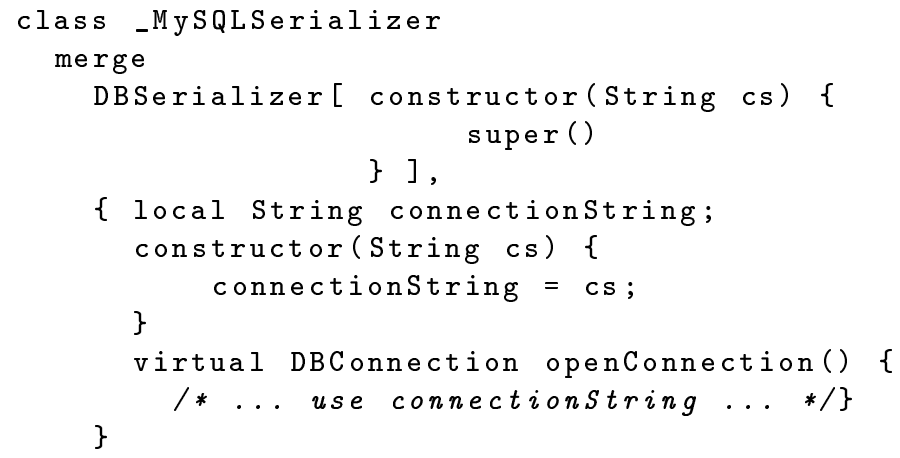

Note the use of the constructor wrapper: the constructor of the anonymous basic class has a String parameter, whereas that of the class DBSerializer, which has no fields, is the default (parameterless) constructor. Hence, a constructor wrapper is inserted, so that the classes we are merging have both a constructor with the same parameters. This allows to create objects of the new class with expressions like new_MySQLSerializer("someConnectionString..."). As mentioned before, the class _MySQLSerializer provides, along the method execute, the method openConnection that we can hide as follows:

class MySQLSerializer

hide openConnection in _MySQLSerializer

Consider now the following class Person, providing a method, named write, to serialize its objects to a database:

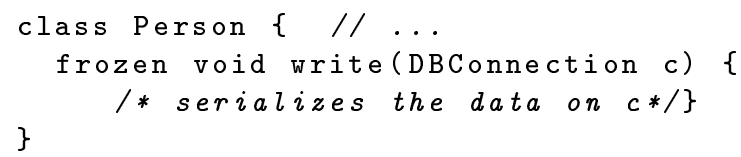

Notwithstanding the inherited method DBSerializer.execute writes the data by invoking the method serialize and not write, using the class Person with MySQLSerializer is not a problem, since we can rename the method before merging the two classes:

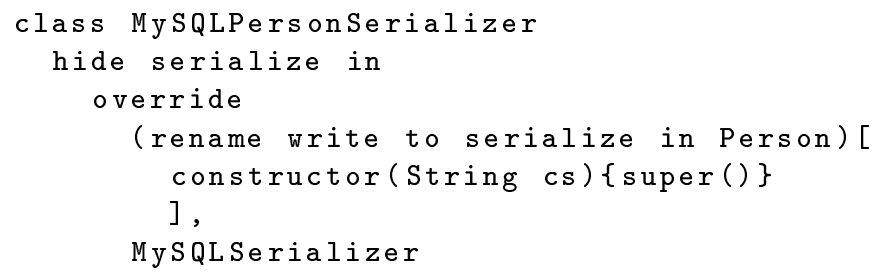

\section{F JIG calculus}

The syntax of the calculus is given in Figure 2. Besides class names, (external) names and variables, we assume an infinite set of internal (member) names $n$. A program consists of two components: a sequence of class declarations (class 
name and class expression), as in FJ, and a sequence of subtype declarations. We assume that no class is declared twice and order is immaterial, hence we can write $p(C)$ for the class expression associated with $C$.

Class expressions $C E$ are basic classes $B$, class names $C$, or are inductively constructed by a set of composition operators. Let us say that $C$ "inherits from" $C^{\prime}$ if the class expression associated with $C$ contains as subterm $C^{\prime}$, or, transitively, $C^{\prime \prime}$ which inherits from $C^{\prime}$. In a well-formed program, we require this generalized inheritance relation to be acyclic, exactly as it is usually required for standard inheritance.

$$
\begin{aligned}
& p \quad::=\overline{c d} \overline{l e q} \\
& \text { cd } \quad::=C \mapsto C E \\
& \text { leq } \quad::=C \leq C^{\prime} \\
& C E \quad::=B|C| \\
& C E_{1}+C E_{2} \quad \text { sum } \\
& { }_{\sigma^{\iota} \mid} C E_{\mid \sigma^{\circ}} \quad \text { reduct } \\
& \text { | freeze }{ }_{N} C E \quad \text { freeze } \\
& \text { | } C E[\mathrm{~K}(\overline{C x})\{\bar{e}\}] C E[\mathrm{TT} \leq C] \\
& \sigma \quad::=\overline{N: T \mapsto N^{\prime}: T^{\prime}}, \overline{-\mapsto N: T} \quad \text { renaming } \\
& N \quad::=F \mid M \quad-\quad \text { external member name } \\
& T \quad::=C \mid M T \quad \text { member type } \\
& M T \quad::=\bar{C} \rightarrow C \quad \text { method type } \\
& B \quad::=[\iota|o| \rho] \\
& \iota \quad::=\overline{n: T \mapsto N} \quad \text { input map } \\
& o \quad::=\overline{N: T \mapsto n} \quad \text { output map } \\
& n \quad::=f \mid m \quad \text { internal member name } \\
& \rho \quad::=\{\tau \bar{\varphi} \kappa \bar{\mu}\} \quad \text { local part } \\
& \tau \quad::=\mathrm{TT} \leq C \\
& \varphi \quad::=C f \\
& \kappa \quad::=\mathrm{K}(\overline{C x})\{\overline{f=e}\} \\
& \mu \quad::=C m(\overline{C x})\{\text { return } e ;\} \\
& e \quad::=x|e . F| e . M(\bar{e})|f| m(\bar{e}) \mid \text { new } C(\bar{e}) \\
& \mid[\bar{\mu} ; v \mid e] \\
& \mid C(\overline{f=e}) \quad \text { (pre-)object } \\
& v, v^{C}::=C(\overline{f=e}) \quad \text { value (object) }
\end{aligned}
$$

Fig. 2. Syntax

Except for some shorter keywords for saving space, the only differences in basic classes w.r.t. the surface syntax given in Figure 1 are the following: 
- There are no modifiers, since their semantics is encoded by distinguishing between external and internal member names, as explained in detail below. This solution is typical of module calculi [19,2], and allows a simpler and intuitive model of composition operators. Internal names are used to refer to class members inside code (method bodies), and can be safely $\alpha$-renamed. On the contrary, external names are used in class composition via operators and in selection of class members by clients.

- Correspondingly, basic classes include, besides previous components which are collected in the local part, an input map from internal to external names, and an output map from external to internal names.

- Expressions include runtime expressions, that is, (pre-)objects and blocks.

Input and output maps are represented as sequences of pairs where the first element has a type annotation. In an input map, internal names which are mapped in the same external name are required to have the same annotation, whereas this is not required in output names, that is, the same member can be exported under different names with different types, see the type system in next section. Renamings $\sigma$ are maps from (annotated) external names into (annotated) external names, represented as sequences of pairs; pairs of form _ $\mapsto N: T$ are used to represent non-surjective maps.

We denote by dom and cod the domain and codomain of a map, respectively. Given a basic class $[\iota|o| \rho]$, with $\rho=\{\tau \bar{\varphi} \kappa \bar{\mu}\}$, we denote by $\operatorname{dom}(\bar{\mu})$ and $\operatorname{dom}(\bar{\varphi})$ the sets of internal names declared in $\bar{\mu}$ and $\bar{\varphi}$, respectively, which are assumed to be disjoint. The union of these two sets, denoted by $\operatorname{dom}(\rho)$, is the set of local names. An internal name $n$ is, instead, abstract if $n \in \operatorname{dom}(\iota), \iota(n) \notin \operatorname{dom}(o)$, and virtual if $\iota(n) \in \operatorname{dom}(o)$. An external name $N$ is abstract if $N \in \operatorname{cod}(\iota) \backslash \operatorname{dom}(o)$, virtual if $N \in \operatorname{cod}(\iota) \cap \operatorname{dom}(o)$, frozen if $N \in \operatorname{dom}(o) \backslash \operatorname{cod}(\iota)$. In a well-formed basic class, local names must be distinct from abstract/virtual internal names, that is, $\operatorname{dom}(\iota) \cap \operatorname{dom}(\rho)=\emptyset$. Moreover, $\operatorname{cod}(o) \subseteq \operatorname{dom}(\rho)$, and, denoting by names $(e)$ the set of internal names in an expression $e$, names $(e) \subseteq \operatorname{dom}(\iota) \cup \operatorname{dom}(\rho)$ for each method body $e$.

A basic class of the surface language can be easily encoded in the calculus as follows. For each member name $N$ we assume (at most) a corresponding external name $N$ and (at most) two internal names $n, n^{\prime}$, depending on the member kind, as detailed below. Client references to $N$ are unaffected, whereas internal references are translated according to the member kind:

- if $N$ is abstract, then there is an association $n \mapsto N$ in the input map, and internal references are translated by $n$,

- if $N$ is virtual, then there is an association $n \mapsto N$ in the input map, an association $N \mapsto n^{\prime}$ in the output map, a definition for $n^{\prime}$ in $\rho$, and internal references are translated by $n$,

- if $N$ is frozen, then there is an association $N \mapsto n^{\prime}$ in the output map, a definition for $n^{\prime}$ in $\rho$, and internal references are translated by $n^{\prime}$.

- if $N$ is local, then there is a definition for $n^{\prime}$ in $\rho$, and internal references are translated by $n^{\prime}$. 
Inside constructor bodies, a field name $F$ on the left-hand side is always translated by $f^{\prime}$ (and all internal accesses/invocations are forbidden in the initialization expressions).

For instance, the class $\mathrm{C}$ shown in the previous section is translated by

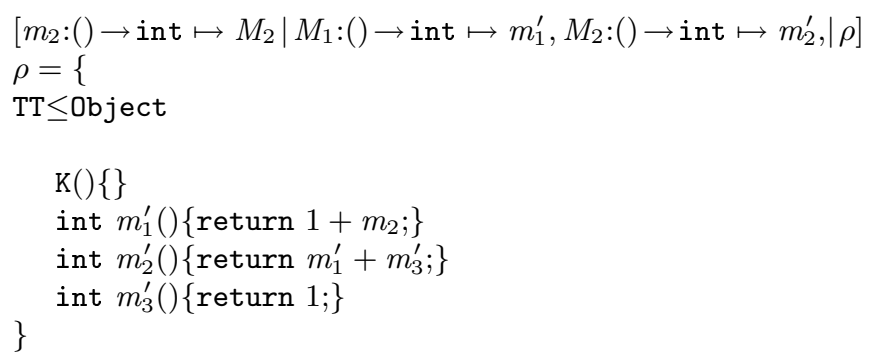

We describe now the two kinds of runtime expressions introduced in the calculus. Expressions of form $C(\overline{f=e})$ denote a pre-object of class $C$, where for each field $f$ there is a corresponding initialization expression. Note the difference with the form new $C(\bar{e})$, which denotes a constructor invocation, whereas in FJ objects can be identified with object creation expressions where arguments are values. As already noted, in FJ it is possible, and convenient, to take this simple and nice solution, since the structure of the instances of a class is globally visible to the whole program. In FJIG, instead, object layout must be hidden to clients, hence constructor parameters have no a priori relation with fields.

Values of the calculus are objects, that is, pre-objects where all initialization expressions are (in turn) values. We use both $v^{C}$ and $v$ as metavariables for values of class $C$, the latter when the class is not relevant.

Moreover, runtime expressions also include block expressions of the form $[\bar{\mu} ; v \mid e]$, which model the execution of $e$ where method internal names are bound in $\bar{\mu}$ and field internal names in the current object $v$. Hence, denoting by $\operatorname{dom}(v)$ the set $\left\{f_{1}, \ldots, f_{n}\right\}$ if $v=C\left(f_{1}=v_{1} \ldots f_{n}=e_{n}\right)$, a block expression is well-formed only if names $(e) \subseteq \operatorname{dom}(\bar{\mu}) \cup \operatorname{dom}(v)$ (hence names $([\bar{\mu} ; v \mid e])=\emptyset$ ) and these two sets are disjoint.

The semantics of an expression $e$ in the context of a program $p$ can be defined in two different ways.

The former, which we call flattening semantics and illustrate in this section, is given in two steps. First, $p$ is reduced to a flat program $p^{\prime}$, that is, a program where every class is basic. To this end, operators are performed and the occurrences of class names are replaced by their defining expressions. Then, $e$ is reduced in the context of $p^{\prime}$. Note that in this case dynamic look-up is always trivial, that is, a class member (e.g., a method) can be always found in the class of the receiver. In next section, we define an alternative direct semantics, where expressions are reduced in the context of non flat programs, hence where dynamic look-up is non trivial.

Flattening rules are defined in the top section of Figure 3. We omit subtype declarations for simplicity since they do not affect semantics. 


$$
\begin{aligned}
& \text { (CDEC1) } \frac{C E \longrightarrow C E^{\prime}}{p, C \mapsto C E \longrightarrow p, C \mapsto C E^{\prime}} \\
& \text { (CDEC2) } \overline{p, C \mapsto B \longrightarrow p[B / C], C \mapsto B} \\
& \text { (suм) } \frac{}{\left[\iota\left|o_{1}\right| \rho_{1}\right]+\left[\iota\left|o_{2}\right| \rho_{2}\right] \longrightarrow\left[\iota\left|o_{1}, o_{2}\right| \rho\right]} \begin{array}{l}
\rho_{i}=\left\{\tau \bar{\varphi}_{i} \mathrm{~K}(\overline{C x})\left\{\bar{f}_{i}\right\} \bar{\mu}_{i}\right\}, i \in\{1,2\} \\
\rho=\left\{\tau \bar{\varphi}_{1}, \bar{\varphi}_{2} \mathrm{~K}(\overline{C x})\left\{\bar{\mu}_{i}=e_{1}, f=e_{2}\right\} \bar{\mu}_{1}, \bar{\mu}_{2}\right\}
\end{array} \\
& \text { (REDuct) } \overline{\left.\sigma^{\iota}|\iota| O \mid \rho\right]_{\mid \sigma^{o}} \longrightarrow\left[\sigma^{\iota} \circ \iota\left|O \circ \sigma^{o}\right| \rho\right]}
\end{aligned}
$$

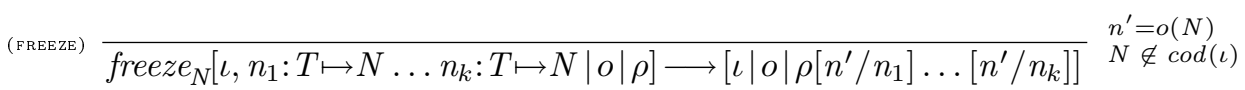

$$
\begin{aligned}
& \text { (Tt wrapping) } \overline{\left[\iota|o|\left\{\mathrm{TT} \leq C^{\prime} \bar{\varphi} \kappa \bar{\mu}\right\}\right][\mathrm{TT} \leq C] \longrightarrow[\iota|o|\{\mathrm{TT} \leq C \bar{\varphi} \kappa \bar{\mu}\}]} \\
& \bar{x}=x_{1} \ldots x_{n}
\end{aligned}
$$

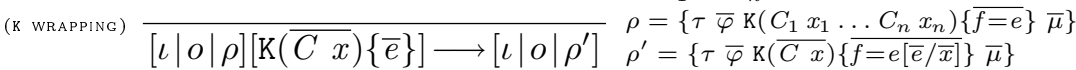

$$
\begin{aligned}
& \text { (стх) } \frac{e \longrightarrow p e^{\prime}}{\mathcal{E}\{e\} \longrightarrow p \mathcal{E}\left\{e^{\prime}\right\}} \quad(\text { (oLIEnt-Field) }) \frac{}{v^{C} . F \longrightarrow p\left[\bar{\mu} ; v^{C} \mid f\right]} \begin{array}{l}
p(C)=[\iota|o|\{\tau \bar{\varphi} \kappa \bar{\mu}\}] \\
o(F)=f
\end{array}
\end{aligned}
$$

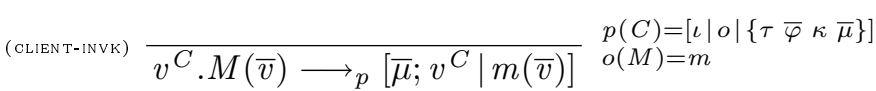

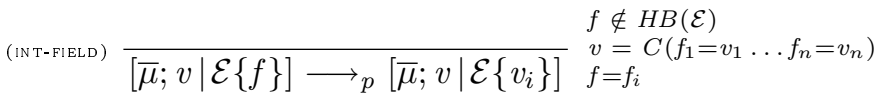

$$
\begin{aligned}
& \text { (InT-InvK) } \overline{\left[\bar{\mu} ; v^{C} \mid \mathcal{E}\{m(\bar{v})\}\right] \longrightarrow p\left[\bar{\mu} ; v^{C} \mid \mathcal{E}\left\{e[\bar{v} / \bar{x}]\left[v^{C} / \text { this }\right]\right\}\right]} \begin{array}{l}
m \notin H B(\mathcal{E}) \\
\bar{\mu}(m)=\langle\bar{x}, \bar{C}, e\rangle
\end{array}
\end{aligned}
$$

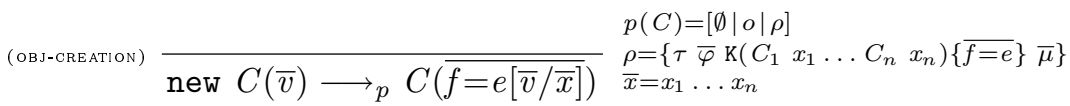

$$
\begin{aligned}
& \text { (Ехіт-вцоск) } \overline{[\bar{\mu} ; v \mid e] \longrightarrow p} e^{n a m e s}(e)=\emptyset
\end{aligned}
$$

Fig. 3. Flattening semantics 
The first two rules define reduction steps of programs, which can be obtained either by reducing one of the class expressions, or, if some class $C$ has already been reduced to a basic class $B$, by replacing by $B$ all occurrences of $C$ as subterms of class expressions.

The remaining rules define reduction steps of class expressions. Rules for sum, reduct and freeze operators are essentially those given in [2], to which we refer for more details. We omit standard contextual closure for brevity.

The expression $o_{1}, o_{2}$ is well-formed only if the two maps have disjoint domains (analogously for other maps). Hence, rule (SUM) can only be applied (implicit side conditions) when the two sets of local $\mathrm{F}$ are disjoint $\left(\operatorname{dom}\left(\rho_{1}\right) \cap \operatorname{dom}\left(\rho_{2}\right)=\emptyset\right)$, as are the sets of output names $\left(\operatorname{dom}\left(o_{1}\right) \cap \operatorname{dom}\left(o_{2}\right)=\emptyset\right)$. The former condition can be always satisfied by an appropriate $\alpha$-conversion, whereas the latter corresponds to a conflict that the programmer can only solve by an explicitly renaming (reduct operator). Input names are required to be the same, and the two constructors are also required to have the same parameters. This is not restrictive since these components can be always made equal by reduct and constructor wrapping operators, respectively.

In rule (REDUCT) the symbol o denotes composition of maps. New input and output names are chosen, modeled by $\operatorname{cod}\left(\sigma^{\iota}\right)$ and $\operatorname{dom}\left(\sigma^{o}\right)$, respectively. Old input names are mapped in new input names by $\sigma^{\iota}$, whereas new output names are mapped into old output names by $\sigma^{o}$. Input names can be shared or added, whereas output names can be duplicated or removed. Composition is well-formed only if type annotations are the same and the annotation of the new name is kept in the resulting map. That is: if $\iota$ contains $n: T \mapsto N$, then $\sigma^{\iota}$ should contain $N: T \mapsto N^{\prime}: T^{\prime}$, and $\sigma^{\iota} \circ \iota$ will contain $n: T^{\prime} \mapsto N^{\prime}$; if $\sigma^{o}$ contains $N^{\prime}: T^{\prime} \mapsto N: T$, then $o$ should contain $N: T \mapsto n$, and $o \circ \sigma^{o}$ will contain $N^{\prime}: T^{\prime} \mapsto n$.

In rule (FREEZE), association from internal names into $N$ are removed from the input map, and occurrences of these names in method bodies are replaced by the local name of the corresponding definition, thus eliminating any dependency on $N$. The second side condition ensures that we actually take all such names.

Rules for constructor and ThisType wrapping just correspond to changing the constructor and the ThisType constraint for a class, respectively.

Reduction rules are given in the second section of Figure 3.

The first rule is the standard contextual closure, where $\mathcal{E}$ denotes a one-hole context and $\mathcal{E}\{e\}$ denotes the expression obtained by filling the hole by $e$.

Client field accesses and method invocations are reduced in two steps. First, they are reduced to a block where the current object is the receiver and the expression to be executed is the corresponding internal field access or method invocation on the name found in the receiver's class; moreover, methods found in the receiver's class are copied into the block and used for resolving further internal method invocations. ${ }^{6}$ Then, the following two rules can be applied.

\footnotetext{
${ }^{6}$ Alternatively, the method body corresponding to an internal name could be again found in the basic class of the receiver; we choose this model because it can be better generalized to direct semantics, see the following.
} 
Signatures are maps from external names into types.

We denote by mtype $(\Delta, C, N)$ the type of member named $N$ in $\Delta(C)$, which is the output type $\mathrm{e}^{7}$ for a defined member, the input type for an abstract member. Internal type environments map internal names to types. Parameter type environments map variables (parameters) into class names. Finally, runtime class type environments map class names to internal type environments.

Typing rules in Figure 5 define the judgments $\vdash p: \Delta$ for programs and $\Delta \vdash$ $C E: C T$ for class expressions.

In (PROG-T), a program has type $\Delta$ if each declared class $C$ has type $\Delta(C)$ w.r.t. $\Delta$, ThisType constraints are satisfied, and declared subtyping relations are safe. The judgment $\Delta \vdash C \leq C^{\prime}$ checks whether $C$ and $C^{\prime}$ are in the reflexive and transitive closure of the subtyping declarations in $\Delta$. The judgment $\Delta \vdash C \leq$ $C^{\prime}$ OK checks whether $C$ is a structural subtype of $C$. The straightforward definition of these judgments is given in the Appendix in Figure 10.

In (BASIC-T), we denote by $\Sigma^{\iota}$ and $\Sigma^{o}$ the signatures extracted from $\iota$ and $o$, respectively; analogously, we denote by $\Gamma^{\iota}, \Gamma^{\bar{\mu}}$ and $\Gamma^{\bar{\varphi}}$ the internal type environments extracted from $\iota, \bar{\mu}$ and $\bar{\varphi}$, respectively.

A basic class is well-typed w.r.t. $\Delta$ under three conditions. First, methods have their declared types w.r.t. $\Delta$, the internal type environment, assigning to member internal names their annotations, and the type in the ThisType constraint (assumed as type for this). Second, the constructor has its declared type w.r.t. $\Delta$ and the internal type environment, assigning to internal field names their annotations. Finally, type annotations in input signature, output signature and local part must be consistent, that is, a virtual member can be used inside the class with a supertype of its exported type (first side condition), and a member can be exported with a subtype of its internal type (second side condition).

Typing rules for sum, reduct and freeze are based on those in [2]. Rule (sumT) imposes the same input signature, constructor type and ThisType constraint, and disjoint output signatures. In (REDUCT-T), the side condition allows a member to be imported with a more specific type, and exported with a more general type. Analogously, rule (THIS-TYPE-T) allows the type of this to become more specific.

Typing rules in Figure 6 define the judgment $\Delta ; \Gamma ; \Pi \vdash e$ : $C$ for well-typed expressions.

They are analogous to FJ rules. However, note that member type is found in receiver's class for client field access and method invocation, whereas it is found in the internal type environment for internal field access and method invocation. Also, note that (NEW-T) requires a class to have an empty input signature in order to be instantiated (see comment to rule (OBJ-CREATION) in previous section).

Finally, typing rules in Figure 7 define the judgment $\Delta ; \Delta^{r} ; \Gamma ; \Pi \vdash e$ : $C$ for welltyped runtime expressions. These expressions are typed using an additional type environment $\Delta^{r}$, which gives for each class the types of its internal field names.

\footnotetext{
7 To provide a richer interface to clients.
} 


$$
\begin{aligned}
& \Delta \vdash C E_{i}: C T_{i} \quad \forall i \in 1 . . n \\
& \Delta \vdash C_{i} \leq C_{i}^{\tau} \forall i \in 1 . . n
\end{aligned}
$$

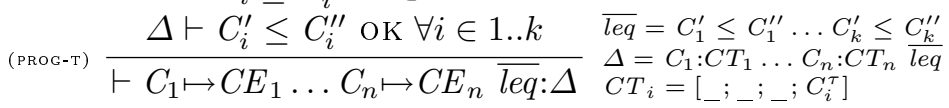

$$
\begin{aligned}
& \text { (cNAME-T) } \frac{}{\Delta \vdash C: C T} \Delta(C)=C T \\
& \Delta ; \Gamma^{\iota}, \Gamma^{\bar{\mu}}, \Gamma^{\bar{\varphi}} ; C \vdash \bar{\mu}: \Gamma^{\bar{\mu}} \\
& \Delta ; \Gamma^{\bar{\varphi}} \vdash \kappa: \bar{C} \quad \Delta \vdash \Sigma^{o}(N) \leq \Sigma^{\iota}(N) \forall N \in \operatorname{dom}(\iota) \cap \operatorname{dom}(o) \\
& \text { (ваAIC-т) } \frac{\Delta ; \Gamma^{\varphi} \vdash \kappa: C}{\Delta \vdash[\iota|o|\{\mathrm{TT} \leq C \bar{\varphi} \kappa \bar{\mu}\}]:\left[\Sigma^{\iota} ; \Sigma^{o} ; \bar{C} ; C\right]} \begin{array}{l}
\Delta \vdash \Sigma^{o}(N) \leq \Sigma^{\iota}(N) \forall N \in \operatorname{dom}(\iota) \cap \operatorname{dom}(o) \\
\Delta \vdash\left(\Gamma^{\bar{\varphi}}, \Gamma^{\bar{\mu}}\right)(o(N)) \leq \Sigma^{o}(N) \forall N \in \operatorname{dom}(o)
\end{array} \\
& \frac{\Delta ; \Gamma ; C \vdash \mu_{i}: M T_{i} \forall i \in 1 . . n}{\Delta ; \Gamma ; C \vdash \bar{\mu}: \Gamma^{\bar{\mu}}} \stackrel{\begin{array}{l}
\bar{\mu}=\mu_{1} \ldots \mu_{n} \\
\Gamma^{\bar{\mu}}=m_{1}: M T_{1} \ldots m_{n}: M T_{n}
\end{array}}{ } \\
& \text { (Метнор-т) } \frac{\Delta ; \Gamma ; \text { this: } C, x_{1}: C_{1} \ldots x_{n}: C_{n} \vdash e: C^{\prime}}{\Delta ; \Gamma ; C \vdash C_{0} m\left(C_{1} x_{1} \ldots C_{n} x_{n}\right)\{\text { return } e ;\}: C_{1} \ldots C_{n} \rightarrow C_{0}} \quad \Delta \vdash C^{\prime} \leq C_{0}
\end{aligned}
$$

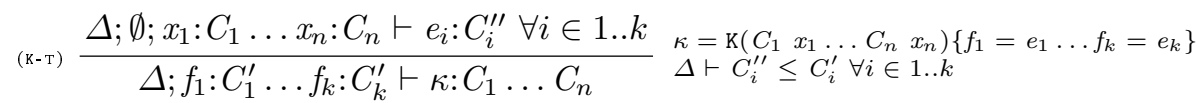

$$
\begin{aligned}
& \Delta \vdash C E_{1}:\left[\Sigma^{\iota} ; \Sigma_{1}^{o} ; \bar{C} ; C\right] \\
& \frac{\Delta \vdash C E_{2}:\left[\Sigma^{\iota} ; \Sigma_{2}^{o} ; \bar{C} ; C\right]}{C E_{1}+C E_{2}:\left[\Sigma^{\iota} ; \Sigma_{1}^{o}, \Sigma_{2}^{o} ; \bar{C} ; C\right]} \operatorname{dom}\left(\Sigma_{1}^{o}\right) \cap \operatorname{dom}\left(\Sigma_{2}^{o}\right)=\emptyset \\
& \text { (REDUCT-T) } \frac{\Delta \vdash C E:\left[\Sigma^{\iota} ; \Sigma^{o} ; \bar{C} ; C\right]}{\Delta \vdash{ }_{\sigma^{\iota} \mid} C E_{\mid \sigma^{o}}:\left[\sigma^{\iota} \circ \Sigma^{\iota} ; \Sigma^{o} \circ \sigma^{o} ; \bar{C} ; C\right]} \Delta \vdash T^{\prime} \leq T \forall N: T \mapsto N^{\prime}: T^{\prime} \in \sigma^{\iota} \cup \sigma^{o} \\
& \underset{(\text { FREEZE-T) }}{ } \frac{\Delta \vdash C E:\left[\Sigma^{\iota}, N: T ; \Sigma^{o} ; \bar{C} ; C\right]}{\Delta \vdash \text { free }^{\prime} e_{N} C E:\left[\Sigma^{\iota} ; \Sigma^{o} ; \bar{C} ; C\right]} \quad N \in \operatorname{dom}\left(\Sigma^{o}\right) \\
& \underset{\text { (TT-WRApPING-T) }}{\Delta \vdash C E[\mathrm{TT} \leq C]:\left[\Sigma^{\iota} ; \Sigma^{o} ; \bar{C} ; C\right]} \quad \Delta \vdash C \leq C^{\prime} \\
& \Delta ; \emptyset ; x_{1}: C_{1} \ldots x_{n}: C_{n} \vdash e_{i}: C_{i}^{\prime \prime} \forall i \in 1 . . k \\
& \text { (K-Wraping-T) } \frac{\Delta \vdash C E:\left[\Sigma^{\iota} ; \Sigma^{o} ; C_{1}^{\prime} \ldots C_{k}^{\prime} ; C\right]}{\Delta \vdash C E\left[\mathrm{~K}\left(C_{1} x_{1} \ldots C_{n} x_{n}\right)\left\{e_{1} \ldots e_{k}\right\}\right]:\left[\Sigma^{\iota} ; \Sigma^{o} ; C_{1} \ldots C_{n} ; C\right]} \Delta \vdash C_{i}^{\prime \prime} \leq C_{i}^{\prime} \forall i \in 1 \ldots k
\end{aligned}
$$

Fig. 5. Typing rules for programs and class expressions 


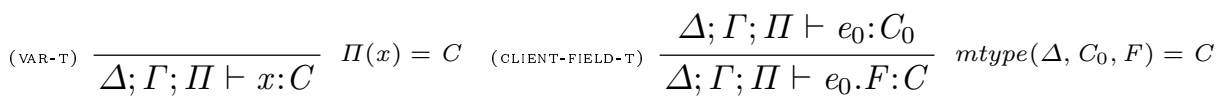

$$
\begin{aligned}
& \Delta ; \Gamma ; \Pi \vdash e_{0}: C_{0}
\end{aligned}
$$

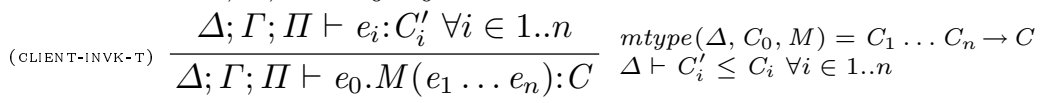

$$
\begin{aligned}
& \text { (Int-Field-t) } \overline{\Delta ; \Gamma ; \Pi \vdash f: C} \Gamma(f)=C
\end{aligned}
$$

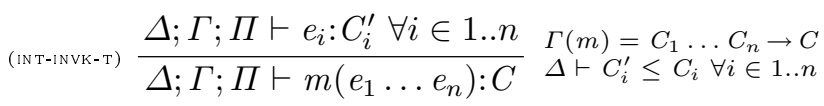

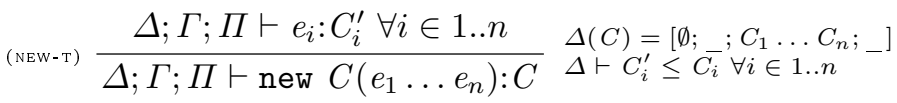

Fig. 6. Typing rules for expressions

Rule (BLOCK-T) checks that the current object is well-typed and the enclosed expression is well-typed in the internal type environment corresponding to the current object's class in $\Delta^{r}$. In this case, the type of the block is that of the enclosed expression. Rule (PRE-OBJ-T) checks that each initialization expressions has a subtype of the type of the corresponding field internal name, found in the internal type environment associated to the (pre)object's class in $\Delta^{r}$. Rules for other forms of expressions are analogous to those in Figure 6, plus propagation of the runtime class type environment.

\begin{tabular}{|c|c|c|}
\hline (вцоск-т) & $\begin{array}{l}\Delta ; \Gamma^{C}, \Gamma^{\bar{\mu}} ; C \vdash \bar{\mu}: \Gamma^{\bar{\mu}} \\
\Delta ; \Delta^{r} ; \Gamma ; \Pi \vdash v: C^{\prime} \\
\Delta ; \Delta^{r} ; \Gamma^{C} ; \Pi \vdash e: C\end{array}$ & \\
\hline (BLOCK-T) & $\Delta ; \Delta^{r} ; \Gamma ; \Pi \vdash[\bar{\mu} ; v \mid e]: C$ & \\
\hline \multirow{2}{*}{ (PRE-OBJ-T) } & $\Delta ; \Delta^{r} ; \Gamma ; \Pi \vdash e_{i}: C_{i}^{\prime} \forall i \in 1 . . n$ & \multirow{2}{*}{$\begin{array}{l}\Delta^{r}(C)=f_{1}: C_{1} \ldots f_{n}: C_{n} \\
\Delta \vdash C_{i}^{\prime} \leq C_{i} \forall i \in 1 . . n\end{array}$} \\
\hline & $\Delta ; \Delta^{r} ; \Gamma ; \Pi \vdash C\left(f_{1}=e_{1} ; \ldots f_{n}=e_{n} ;\right): C$ & \\
\hline
\end{tabular}

Fig. 7. Typing rules for runtime expressions

Soundness of the type system is expressed by the following theorems. 
Theorem 1 (Soundness w.r.t. flattening relation). If $\vdash p: \Delta$, then $p \stackrel{\star}{\longrightarrow} p^{\prime}$ for some $p^{\prime}$ flat program, and $\vdash p^{\prime}: \Delta$.

Proof. The proof is a simple adaptation of that given in [2].

Let us denote by $\Delta_{p}^{r}$ the runtime class type environment extracted from a flat program $p$. That is, for each instantiable basic class declaration

$C \mapsto[\emptyset|o|\{\tau \bar{\varphi} \kappa \bar{\mu}\}]$ in $p, \Delta_{p}^{r}(C)=\Gamma^{\bar{\varphi}}$.

Theorem 2 (Progress). If $\vdash p: \Delta$ and $\Delta ; \Delta_{p}^{r} ; \emptyset ; \emptyset \vdash e$ : $C$, then either $e$ is a value or $e \longrightarrow_{p} e^{\prime}$ for some $e^{\prime}$.

Theorem 3 (Subject reduction). If $\vdash p: \Delta, \Delta ; \Delta_{p}^{r} ; \Gamma ; \Pi \vdash e: C$, and $e \longrightarrow p$ $e^{\prime}$, then $\Delta ; \Delta_{p}^{r} ; \Gamma ; \Pi \vdash e: C^{\prime}$, and $\Delta \vdash C^{\prime} \leq C$.

\section{Direct semantics}

Direct semantics allows a modular approach where each class (module) can be analyzed (notably, compiled) in isolation, since references to other classes do not need to be resolved before runtime. In this case, look-up is a non trivial procedure where a class member (e.g., method) is possibly retrieved from other classes and modified as effect of the module operators.

In order to define direct semantics, block expressions are generalized as shown in the top section of Figure 8. That is, besides the previous components, a block contains a path map which maps internal names to paths $\pi$, which denote a subterm in the class expression defining the class $C$ of the current object (an implementation could use a pointer). More precisely, a path $\pi$ always denotes a subterm of the form freeze ${ }_{N} C E$, and is used as a permanent reference to the definition of member $N$ in $C E$. Indeed, the external name $N$ can be changed or removed by effect of outer reduct operators; however, references via $\pi$ are not affected. Hence, when a reference $\pi$ is encountered during current method execution, lookup of $N$ in $C E$ is triggered (see more explanations below). In flattening semantics, $C$ is always a basic class, hence this case never happens. A generalized block expression $[\hat{\imath} ; \bar{\mu} ; v \mid e]$ is well-formed only if names $(e) \subseteq \operatorname{dom}(\hat{\imath}) \cup \operatorname{dom}(\bar{\mu}) \cup \operatorname{dom}(v)$ and these three sets are disjoint.

The center section of the figure contains the new rules for expression reduction. When a member reference (external name or path) $\hat{N}$ needs to be resolved, the lookup procedure starts the search of $\hat{N}$ from receiver's class $C$ and, if successful, returns a corresponding internal name inside a block expression, as shown in rules (CLIENT-FIELD) and (CLIENT-INVK). In flattening semantics, $C$ is always a basic class, hence lookup is trivial and the side condition can be equivalently expressed as in the analogous rules in Figure 3.

When an internal name $n$ is encountered, it is either directly mapped to a definition, or to a path. The former case happens when $n$ was a local name in the basic class containing the definition of the method which is currently being executed. In this case, the corresponding definition is taken, as shown in rules 


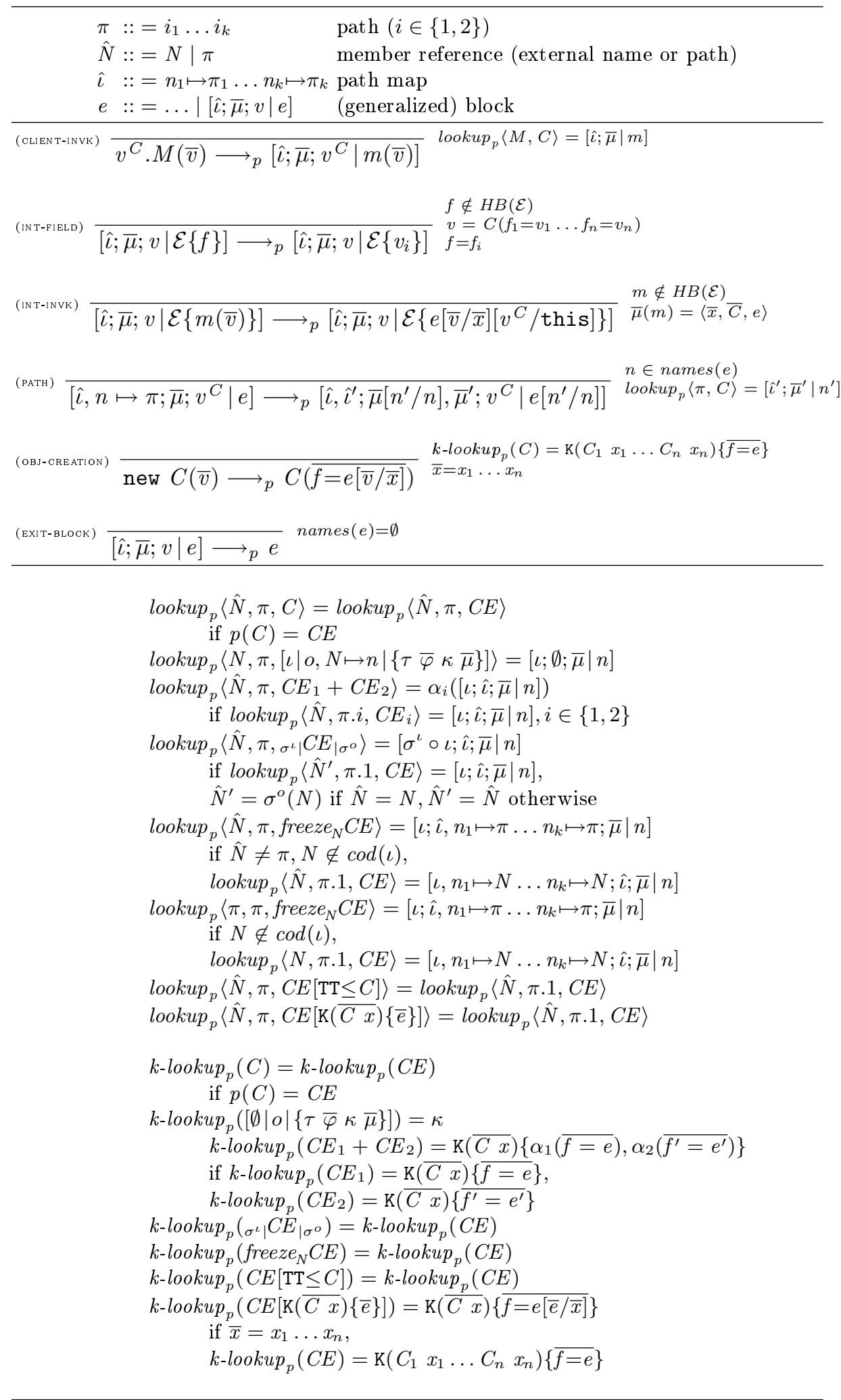

Fig. 8. Direct semantics 
(INT-FIELD) and (INT-INVK). The latter case happens when $n$ was an abstract or virtual name inside the basic class containing the definition of the method which is currently executed, and $n$ has been permanently bound to some definition by an outer freeze operator (recall that only classes where all members are frozen can be instantiated). In this case, lookup of this definition is started from receiver's class via the path $\pi$, and, if successful, the internal name $n$ is replaced by the name $n^{\prime}$ found by lookup; moreover, the corresponding path map and methods are merged with the original ones ( $\alpha$-renaming can be used to avoid conflicts among internal names in this phase). This is shown in rule (PATH). In flattening semantics, the latter case never happens, hence only the first two rules are needed.

Creation of an instance of class, say, $C$, also involves a constructor lookup procedure, which returns, starting from class $C$, the appropriate constructor, possibly by retrieving and modifying constructors of other classes (this generalizes what happens in standard Java-like languages, where the superclass constructor is always invoked). In flattening semantics, $C$ is always a basic class, hence constructor lookup is trivial and the side condition can be equivalently expressed as in the corresponding rule in Figure 3.

The remaining rule is analogous to that given for the flattening case.

Lookup and constructor lookup are defined in the bottom section of the figure. The lookup procedure is modeled by a function which, given a program $p$, takes three more arguments: a member reference (external name or path) $\hat{N}$, a path $\pi$, which acts as an accumulator and keeps track of the current subterm of the class expression which is examined, and a class name $C$. When lookup is started, $\pi$ is always the empty path $\Lambda$, and $\operatorname{lookup}_{p}\langle\hat{N}, \Lambda, C\rangle$ is abbreviated by $\operatorname{lookup}_{p}\langle\hat{N}, C\rangle$.

The lookup function returns a triple consisting of input map, path map, methods and an internal name, written $[\iota ; \hat{\imath} ; \bar{\mu} \mid n]$. However, the final result of lookup (that is, the result returned for the initial call) is expected to be always of form $[\emptyset ; \hat{\imath} ; \bar{\mu} \mid n]$, abbreviated by $[\hat{\iota} ; \bar{\mu} \mid n]$, since all abstract/virtual internal names are expected to be eventually bound to a path as effect of some freeze operator.

The first two clauses defining lookup are trivial and state that looking for a member reference starting from a class name $C$ means looking in the definition of $C$, and that looking for an external name $N$ in a basic class only succeeds if the name is present in the class, and returns the corresponding input map, methods and internal name. Note that the case where we look for a path $\pi$ in a basic class is expected to never happen.

The third clause defines lookup on a sum expression. In this case, lookup is propagated to both arguments. This definition is a priori non-deterministic, but is expected to be deterministic on class expressions which can be safely flattened, since in this case an external name cannot be found on both sides. For member references which are paths, instead, determinism is guaranteed by construction since the path exactly corresponds to a subterm. In case lookup succeeds on one of the two arguments, the result is modified by renaming field local names in a way which keeps track of this argument. For instance, if lookup succeeded on the 
first argument, then every field internal name $f$ is renamed to $f .1$. This renaming is denoted by $\alpha_{i}$. We choose this canonical $\alpha$-renaming for concreteness, but any other could be chosen, provided that it is consistent with that in constructor lookup.

For instance, let us consider the following program (assuming integer values and operations to be available, in order to write more readable examples):

$$
\begin{aligned}
C \mapsto & C_{1}+C_{2} \\
C_{1} \mapsto & {[\emptyset|\ldots|\{\text { int } f ; \mathrm{K}()\{f=3\} \quad \ldots\}] } \\
C_{2} \mapsto & {[\emptyset|\ldots, M \mapsto m|} \\
& \{\tau \text { int } f ; \mathrm{K}()\{f=5\} \text { int } m() \text { \{return } f+1 ;\}\}]
\end{aligned}
$$

and the expression new $C() \cdot M()$. An instance of class $C$ has two fields, inherited from $C_{1}$ and $C_{2}$, and initialized to 3 and 5 , respectively. They are both named $f$ in the original classes; however, they are renamed during constructor lookup (see the clause for sum), hence the above expression reduces to $C(f .1 \mapsto 3, f .2 \mapsto 5) . M()$. Now, $M$ is invoked, starting the lookup from $C$, and the search is propagated to both $C_{1}$ and $C_{2}$. Only the lookup in $C_{2}$ is successful and returns the result

$$
[; ; \text { int } m()\{\text { return } f+1 ;\} \mid m]
$$

which is modified in $[;$; int $m()\{$ return $f .2+1 ;\} \mid m]$ to take into account that the method has been found in the second argument. Hence, this method invocation reduces to [; int $m()\{$ return $f .2+1 ;\} ; C(f .1 \mapsto 3, f .2 \mapsto 5) \mid m]$ where the body of $m$ correctly refers to the second field.

In flattening semantics, $C$ reduces to the following basic class:

$$
\begin{aligned}
& {[\emptyset|\ldots, M \mapsto m| \rho]} \\
& \rho=\{\tau \text { int } f .1 ; \text { int } f .2 ; \kappa \text { int } m()\{\text { return } f .2+1 ;\} \ldots\} \\
& \kappa=\mathrm{K}()\{f .1=3, f .2=5\}
\end{aligned}
$$

Note that here the clash between the two fields is resolved during flattening (hence before runtime), by $\alpha$-renaming. We have chosen as $\alpha$-renaming the same used in direct semantics as an help for the reader, but of course in this case any other arbitrary $\alpha$-renaming would work as well.

The fourth clause defines lookup on a reduct expression. In this case, lookup of an external name is propagated under the name the member has in the argument, given by the output renaming $\sigma^{o}$. Instead, lookup of a path is simply propagated, since paths are permanent references which are not affected by renamings. Moreover, the result of lookup on the argument must be modified to ensure that internal names refer to the appropriate external names obtained via the input renaming $\sigma^{\iota}$.

For instance, consider a program including

$$
\begin{aligned}
& C \mapsto{ }_{M_{1} \mapsto M^{\prime} \mid} C_{\mid M \mapsto M^{\prime}}^{\prime} \\
& C^{\prime} \mapsto\left[m^{\prime} \mapsto M_{1}\left|M^{\prime} \mapsto m\right|\left\{\ldots \text { int } m()\left\{\text { return } m^{\prime}() ;\right\}\right\}\right]
\end{aligned}
$$


and assume that some method invocation triggers the lookup for $M$ in $C$. Then, the lookup is propagated under the name $M^{\prime}$ to $C^{\prime}$. The lookup of $M^{\prime}$ in $C^{\prime}$ is successful and returns the result $\left[m^{\prime} \mapsto M_{1} ;\right.$; int $m()\left\{\right.$ return $\left.\left.m^{\prime}() ;\right\} \mid m\right]$ which is modified in $\left[m^{\prime} \mapsto M_{1}^{\prime} ;\right.$; int $m()$ \{return $\left.\left.m^{\prime}() ;\right\} \mid m\right]$ as an effect of the input renaming.

In flattening semantics, $C$ reduces to the following basic class:

$$
\left.\left[m^{\prime} \mapsto M_{1}^{\prime}|M \mapsto m|\left\{\ldots \text { int } m() \text { \{return } m^{\prime}() ;\right\}\right\}\right]
$$

There are two clauses defining lookup on a freeze expression. The former handles most cases, except the special situation in which we are exactly looking for the member that has been frozen in the current subterm $\pi$, which has the form freez $_{N} C E$. In this special case (second clause) the lookup of $N$ in $C E$ is triggered. Moreover, the result is modified, since internal names referring to $N$ must now refer to the permanent reference $\pi$. Otherwise (first clause), the lookup is propagated, and the result of the lookup on the argument is modified as in the previous case.

The following example illustrates the second clause. Consider the program

$$
\begin{aligned}
C \mapsto & \text { freeze }_{F} C^{\prime} \\
C^{\prime} \mapsto & {\left[f \mapsto F\left|F \mapsto f^{\prime}, M \mapsto m\right|\right.} \\
& \left.\left.\left\{\text { int } f^{\prime} ; \mathrm{K}()\left\{f^{\prime}=42\right\} \text { int } m() \text { \{return } f+1 ;\right\}\right\}\right]
\end{aligned}
$$

and the expression new $C() \cdot M()$.

An instance of class $C$ has one field, inherited from $C^{\prime}$ and initialized to 42 . Hence, the above expression reduces to $C\left(f^{\prime} \mapsto 42\right) . M()$. Now, $M$ is invoked, starting the lookup from $C$, and the search is propagated to $C^{\prime}$. The lookup in $C^{\prime}$ is successful and returns the result $[f \mapsto F$; int $m()$ \{return $\left.f+1 ;\} \mid m\right]$, which is modified in $[; f \mapsto \Lambda$; int $m()\{$ return $f+1 ;\} \mid m]$, where $\Lambda$ denotes the empty path, to take into account that $F$ has been frozen. Hence, the method invocation reduces to $[f \mapsto \Lambda$; int $m()$ rreturn $f+1 ;\} ; C(f \mapsto 42) \mid m]$, where the body of $m$ correctly refers to $F$ frozen in the top level freeze.

In flattening semantics, $C$ reduces to the following basic class:

$$
\left[\emptyset\left|F \mapsto f^{\prime}, M \mapsto m\right|\left\{\text { int } f^{\prime} ; \mathrm{K}()\left\{f^{\prime}=42\right\} \text { int } m()\left\{\text { return } f^{\prime}+1 ;\right\}\right\}\right]
$$

Figure 9 shows a more involved example comparing flattening and direct semantics.

The top section of the figure lists some abbreviations, the second shows the four classes composing program $p$. Class $A$ defines the frozen method $M$ whose body invokes the abstract method $M^{\prime}$. Class $B$ has one local field $f$ initialized to 0 and defines the frozen method $M^{\prime}$ which returns this field. Class $C$ is obtained by summing $A$ and $B$, and then freezing method $M^{\prime}$. Finally, class $D$ is obtained by hiding method $M^{\prime}$ in $C$ (in the reduct, the input renaming is empty since there are no input names, and the output renaming maps "no new name" into $M^{\prime}$ and is the identity on $M$ ) and then summing a new definition for $M^{\prime}$. The following three sections of the figure shows how the class expressions for $C$ and $D$ are reduced, the resulting flat program $p^{\prime}$ and the reduction of expression 


\begin{tabular}{|c|c|}
\hline \multicolumn{2}{|c|}{$\begin{array}{l}v^{D} \equiv D(f .2 .1=0) \\
\mu \equiv C m()\left\{\text { return } m^{\prime}() ;\right\} \\
\mu^{\prime \prime} \equiv C m^{\prime \prime}()\{\text { return } f .2 .1 ;\} \\
\bar{\mu}_{D} \equiv C m()\left\{\text { return } m^{\prime \prime}() ;\right\} ; C m^{\prime \prime}()\{\text { return } f .2 .1 ;\} ; C m^{\prime \prime \prime}()\{\text { return } 8 ;\} \\
\bar{\mu}_{\text {sum }} \equiv C m()\left\{\text { return } m^{\prime}() ;\right\} ; C m^{\prime \prime}()\{\text { return } f .2 ;\} \\
\bar{\mu}_{C} \equiv C m()\left\{\text { return } m^{\prime \prime}() ;\right\} ; C m^{\prime \prime}()\{\text { return } f .2 ;\}\end{array}$} \\
\hline \multicolumn{2}{|c|}{$\begin{aligned} p \equiv A & =\left[m^{\prime} \mapsto M^{\prime}|M \mapsto m|\left\{\mathrm{K}()\{\} C m()\left\{\text { return } m^{\prime}() ;\right\}\right\}\right] \\
B & =\left[\emptyset\left|M^{\prime} \mapsto m^{\prime}\right|\left\{C f ; \mathrm{K}()\{f=0\} C m^{\prime}()\{\text { return } f ;\}\right\}\right] \\
& C=f r e e z e_{M^{\prime}}(A+B) \\
& D={ }_{\emptyset \mid} C_{\mid} \mapsto M^{\prime}, M \mapsto M \\
& \end{aligned}$} \\
\hline \multirow{2}{*}{\multicolumn{2}{|c|}{ 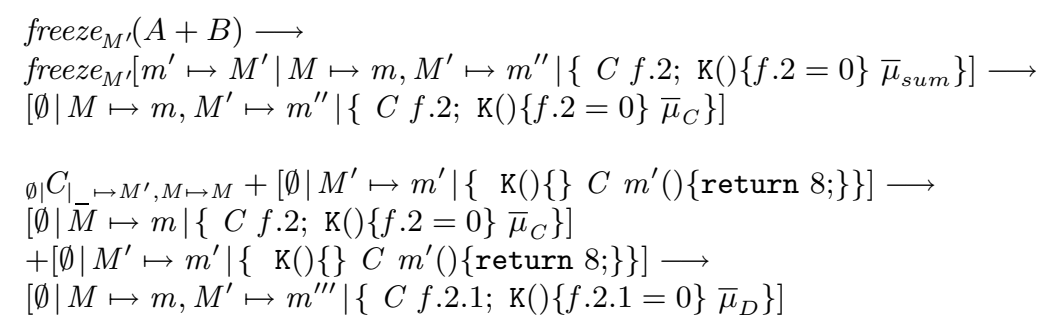 }} \\
\hline & \\
\hline \multicolumn{2}{|c|}{$\begin{aligned} p^{\prime} \equiv A & =\left[m^{\prime} \mapsto M^{\prime}|M \mapsto m|\left\{\mathrm{K}()\{\} C m()\left\{\text { return } m^{\prime}() ;\right\}\right\}\right] \\
B & =\left[\emptyset\left|M^{\prime} \mapsto m^{\prime}\right|\left\{C f ; \mathrm{K}()\{f=0\} C m^{\prime}()\{\text { return } f ;\}\right\}\right] \\
C & =\left[\emptyset\left|M \mapsto m, M^{\prime} \mapsto m^{\prime \prime}\right|\left\{C f .2 ; \mathrm{K}()\{f .2=0\} \bar{\mu}_{C}\right\}\right] \\
D & =\left[\emptyset\left|M \mapsto m, M^{\prime} \mapsto m^{\prime \prime \prime}\right|\left\{C f .2 .1 ; \mathrm{K}()\{f .2 .1=0\} \bar{\mu}_{D}\right\}\right]\end{aligned}$} \\
\hline \multicolumn{2}{|c|}{$\begin{array}{l}\text { new } D() \cdot M() \longrightarrow p_{p^{\prime}} v^{D} \cdot M() \longrightarrow{ }_{p^{\prime}}\left[\bar{\mu}_{D} ; v^{D} \mid m()\right] \longrightarrow p^{\prime}\left[\bar{\mu}_{D} ; v^{D} \mid m^{\prime \prime}()\right] \longrightarrow p^{\prime} \\
{\left[\bar{\mu}_{D} ; v^{D} \mid f \cdot 2.1\right] \longrightarrow p^{\prime}\left[\bar{\mu}_{D} ; v^{D} \mid 0\right] \stackrel{p^{\prime}}{ }=}\end{array}$} \\
\hline $\begin{array}{l}\text { new } D() \cdot M() \longrightarrow p \\
v^{D} \cdot M() \longrightarrow p \\
{\left[m^{\prime} \mapsto 1.1 ; \mu ; v^{D} \mid m()\right] \longrightarrow p} \\
{\left[m^{\prime} \mapsto 1.1 ; \mu ; v^{D} \mid m^{\prime}()\right] \longrightarrow p} \\
{\left[m^{\prime} \mapsto 1.1 ; \mu ; \mu^{\prime \prime} ; v^{D} \mid m^{\prime \prime}()\right] \longrightarrow p} \\
{\left[m^{\prime} \mapsto 1.1 ; \mu ; \mu^{\prime \prime} ; v^{D} \mid f .2 .1\right] \longrightarrow p} \\
{\left[m^{\prime} \mapsto 1.1 ; \mu ; \mu^{\prime \prime} ; v^{D} \mid 0\right] \longrightarrow p} \\
0\end{array}$ & $\begin{array}{l}\text { k-lookup }(D)=\mathrm{K}()\{f .2 .1=0\} \\
\text { lookup }_{p}\langle M, \Lambda, D\rangle=\left[\Lambda ; m^{\prime} \mapsto 1.1 ; \mu \mid m\right] \\
\text { lookup }_{p}\langle 1.1, \Lambda, D\rangle=\left[\Lambda ; \Lambda ; \mu^{\prime \prime} \mid m^{\prime \prime}\right]\end{array}$ \\
\hline
\end{tabular}

Fig. 9. Example 
new $D() . M()$ in the context of $p^{\prime}$. Finally, the last section shows direct semantics of the same expression in the context of $p$.

The example shows how the method originally called $M^{\prime}$ in $B$ is correctly invoked via the path 1.1, even though $M^{\prime}$ has been hidden and then replaced by an homonymous method.

The following theorem states that flattening is equivalent to direct semantics. We denote by $\stackrel{\star}{\longrightarrow}$ the reflexive and transitive closure of the flattening relation, and analogously for the reduction relation. The proof can be found in [15].

Theorem 4. If $p \stackrel{\star}{\longrightarrow} p^{\prime}$, then $e \stackrel{\star}{\longrightarrow} p$ iff $e \stackrel{\star}{\longrightarrow} p^{\prime} v$.

\section{Conclusion}

We have presented FJIG, a core calculus which formalizes the Bracha's Jigsaw framework [7] in a Java-like setting. The design of F JIG comes out naturally, yet not trivially, by taking Featherweight Java [14] as starting point and replacing inheritance by the more general composition operators of Jigsaw.

We believe that such a core calculus can be useful for many research directions. First, it provides a simple unifying formalism for encoding and comparing a large variety of different mechanisms for software composition in class-based languages, including standard inheritance, mixin classes, traits and hiding. Then, it can serve as the basis for the design of a real language based on Jigsaw principles. Moreover, it could be enriched by behavioural types, leading to a classbased specification language, in the spirit of, e.g., JML [16], allowing modular development and composition of class specifications.

We have also defined two different execution models for the calculus, flattening and direct semantics, and proved their equivalence. That is, we have shown the equivalence of two different views on inheritance in a formal setting with a more sophisticated composition mechanism, where, e.g., mixin classes and traits can be subsumed. This can also greatly help in integrating such features, or other modularity mechanisms, in standard class-based languages, since it gives practical hints on implementation.

Apart from the two key references mentioned above, this work has been directly influenced by work on traits $[18,9]$, mostly by the recent developments $[17,5,6]$. In particular, we share with $[5,6]$ the objective of replacing inheritance by more flexible operators. Concerning flattening and direct semantics, the most direct source of inspiration for our work has been [17], which defines a direct semantics for traits. Essentially, their dynamic look-up algorithm can be seen as a simplified version, handling sum and output reduct only, of ours.

The focus of this paper is on providing a simple and compact model for a language based on the Jigsaw framework in a Java-like setting, hence we have only outlined in Section 1 a simple surface language. As mentioned above, we leave to further work a deeper investigation of a realistic language design, and a more precise analysis on how different mechanisms such as standard inheritance, mixin classes, traits can be encoded into F JIG. We also plan to develop a prototype implementation; a very preliminary interpreter of flattening semantics, assigned as 
master thesis, can be found at http: //www . disi.unige.it/person/LagorioG/FJig/. We also plan to investigate smart implementation techniques of direct semantics in the prototype interpreter.

\section{References}

1. Davide Ancona, Giovanni Lagorio, and Elena Zucca. Jam-designing a Java extension with mixins. ACM Transactions on Programming Languages and Systems, 25(5):641-712, September 2003.

2. Davide Ancona and Elena Zucca. A calculus of module systems. Journ. of Functional Programming, 12(2):91-132, 2002.

3. Alexandre Bergel, Stéphane Ducasse, Oscar Nierstrasz, and Roel Wuyts. Stateful traits. In Advances in Smalltalk - 14th International Smalltalk Conference (ISC 2006), volume 4406, pages 66-90. Springer, 2007.

4. Alexandre Bergel, Stéphane Ducasse, Oscar Nierstrasz, and Roel Wuyts. Stateful traits and their formalization. Comput. Lang. Syst. Struct., 34(2-3):83-108, 2008.

5. Viviana Bono, Ferruccio Damiani, and Elena Giachino. Separating type, behavior, andstate to achieve very fine-grained reuse. In 9th Intl. Workshop on Formal Techniques for Java-like Programs, 2007.

6. Viviana Bono, Ferruccio Damiani, and Elena Giachino. On traits and types in a Java-like setting. In TCS'08 - IFIP Int. Conf. on Theoretical Computer Science. Springer, 2008.

7. Gilad Bracha. The Programming Language JIGSAW: Mixins, Modularity and Multiple Inheritance. PhD thesis, Department of Comp. Sci., Univ. of Utah, 1992.

8. D. Duggan and C. Sourelis. Mixin modules. In Intl. Conf. on Functional Programming 1996, pages 262-273. ACM Press, 1996.

9. Kathleen Fisher and John Reppy. A typed calculus of traits. In FOOL'04 - Intl. Workshop on Foundations of Object Oriented Languages, 2004.

10. Matthew Flatt, Shriram Krishnamurthi, and Matthias Felleisen. Classes and mixins. In ACM Symp. on Principles of Programming Languages 1998, pages 171-183. ACM Press, 1998.

11. Erich Gamma, Richard Helm, Ralph E. Johnson, and John M. Vlissides. Design Patterns: Elements od Reusable Object-Oriented Software. Addison-Wesley Professional Computing Series. Addison-Wesley, 1995.

12. Tom Hirschowitz and Xavier Leroy. Mixin modules in a call-by-value setting. In ESOP 2002 - European Symposium on Programming 2002, number 2305 in LNCS, pages 6-20. Springer, 2002.

13. Tom Hirschowitz, Xavier Leroy, and J. B. Wells. Call-by-value mixin modules: Reduction semantics, side effects, types. In ESOP 2003 - European Symposium on Programming 2003, number 2986 in LNCS, pages 64-78. Springer, 2004.

14. Atsushi Igarashi, Benjamin C. Pierce, and Philip Wadler. Featherweight Java: a minimal core calculus for Java and GJ. ACM Transactions on Programming Languages and Systems, 23(3):396-450, 2001.

15. Giovanni Lagorio, Marco Servetto, and Elena Zucca. Flattening versus direct semantics for Featherweight Jigsaw. In FOOL'09 - Intl. Workshop on Foundations of Object Oriented Languages, 2009. To appear.

16. Gary T. Leavens. Tutorial on JML, the Java modeling language. In Automated Software Engineering (ASE 2007). ACM Press, 2007. 
17. Luigi Liquori and Arnaud Spiwack. FeatherTrait: A modest extension of Featherweight Java. ACM Transactions on Programming Languages and Systems, 30(2), 2008.

18. Nathanael Schärli, Stéphane Ducasse, Oscar Nierstrasz, and Andrew P. Black. Traits: Composable units of behaviour. In ECOOP'03 - Object-Oriented Programming, volume 2743 of $L N C S$, pages 248-274. Springer, 2003.

19. J. B. Wells and R. Vestergaard. Confluent equational reasoning for linking with first-class primitive modules. In ESOP 2000 - European Symposium on Programming 2000, number 1782 in LNCS, pages 412-428. Springer, 2000.

\section{A Subtyping relations}

$$
\begin{aligned}
& \text { (structural-sub) } \frac{}{\Delta \vdash C \leq C^{\prime} \mathrm{OK}} \operatorname{mtype}\left(\Delta, C^{\prime}, N\right)=T^{\prime} \Rightarrow \operatorname{mtype}(\Delta, C, N)=T, \Delta \vdash T \leq T^{\prime} \\
& \Delta \vdash C_{i}^{\prime} \leq C_{i} \quad \forall i \in 1 . . n \\
& \text { (метнод-suв) } \frac{\Delta \vdash C \leq C^{\prime}}{\Delta \vdash C_{1} \ldots C_{n} \rightarrow C \leq C_{1}^{\prime} \ldots C_{n}^{\prime} \rightarrow C^{\prime}} \quad \text { (RefL-sub) } \frac{}{\Delta \vdash C \leq C} \quad \Delta(C)= \\
& \text { (Decl-sub) } \frac{\Delta \vdash C C^{\prime}}{\Delta \vdash C C^{\prime} \in \Delta \quad{ }_{\text {(trans-s) }}} \frac{\Delta \vdash C_{1} \leq C_{2} \quad \Delta \vdash C_{2} \leq C_{3}}{\Delta \vdash C_{1} \leq C_{3}}
\end{aligned}
$$

Fig. 10. Subtyping relationship

\section{B Proofs}

Lemma 1. If $\Delta ; \Delta^{r} ; \Gamma ; \Pi \vdash e: C^{\prime}$, names $(e)=\emptyset$ and $e$ does not contain free variables (parameter names), then $\Delta ; \Delta^{r} ; \emptyset ; \emptyset \vdash e: C^{\prime}$.

\section{Lemma 2.}

$i:$ If $\Delta ; \Delta^{r} ; \Gamma ; \Pi \vdash \mathcal{E}\{e\}: C^{\prime}$, then, for some $C, \Delta ; \Delta^{r} ; \Gamma^{\prime} ; \Pi^{\prime} \vdash e: C$.

ii: If $\Delta ; \Delta^{r} ; \Gamma ; \Pi \vdash \mathcal{E}\{e\}: C^{\prime}$ and $H B(\mathcal{E})=\emptyset$, then, for some $C, \Delta ; \Gamma ; \Pi \vdash e: C$.

Lemma 3. If $\vdash p: \Delta$, then
$i: \operatorname{dom}(p)=\operatorname{dom}(\Delta)$
ii: for all $C \in \operatorname{dom}(p)$
$A: \Delta(C)=\left[\Sigma^{\iota} ; \Sigma^{o} ; \bar{C} ; C\right]$
$B: p(C)=[\iota|o|\{\tau \bar{\varphi} \kappa \bar{\mu}\}]$
$C: \operatorname{dom}(\iota)=\operatorname{dom}\left(\Sigma^{\iota}\right)$
D: $\operatorname{dom}(o)=\operatorname{dom}\left(\Sigma^{o}\right)$ 
$E: \#(\bar{x})=\#(\bar{C})$

$F: \Delta \vdash p(C): \Delta(C)$

Lemma 4. For all $\Delta, C, N$, mtype $(\Delta, C, N)=T$ iff $\left(\Delta(C)=\left[\Sigma^{\iota} ; \Sigma^{o}, N \mapsto\right.\right.$ $T ; \bar{C} ; C]$ or $\left.\Delta(C)=\left[\Sigma^{\iota}, N \mapsto T ; \Sigma^{o} ; \bar{C} ; C^{\prime}\right]\right)$.

Lemma 5. If $\Delta ; \Delta^{r} ; \Gamma ; \Pi \vdash v^{C}: C$ and mtype $(\Delta, C, N)=T$, then

$i: \Delta(C)=\left[\emptyset ; \Sigma^{o}, N \mapsto T ; \bar{C} ; C^{\prime}\right]$

ii: $p(C)=[\emptyset|o, N \mapsto n| \rho]$

\section{Lemma 6.}

$i: \Delta ; \Delta^{r} ; \Delta^{r}(C) ; \Pi \vdash f: C^{\prime} \Delta ; \Delta^{r} ; \emptyset ; \emptyset \vdash C\left(f_{1}=v_{1} \ldots f_{n}=v_{n}\right): C$ implies $f=$ $f_{i}, \Delta ; \Delta^{r} ; \Delta^{r}(C) ; \Pi \vdash v_{i}: C^{\prime \prime}$ and $\Delta \vdash C^{\prime \prime} \leq C^{\prime}$.

ii: $\Delta ; \Delta^{r} ; \Gamma^{\prime} ; \Pi^{\prime} \vdash\left[; v^{C^{\prime}} \mid m(\bar{e})\right]: C$ and $\Delta^{r}\left(\bar{C}^{\prime}\right)(m)=\bar{C} \rightarrow C$ implies \# $(\bar{e})=$ $\#(\bar{x})$.

Lemma 7. Any expression $e$ which is not a value is of the form $e=\mathcal{E}\left\{e^{\prime}\right\}$, with:

$-e^{\prime}=x$ or

$-e^{\prime}=\left[\bar{\mu} ; v \mid e^{\prime \prime}\right]$ or

$-e^{\prime}=f$ or

$-e^{\prime}=m(\bar{v})$ or

$-e^{\prime}=v \cdot F$ or

$-e^{\prime}=v \cdot M(\bar{v})$ or

$-e^{\prime}=$ new $C((\bar{v}))$.

Theorem 5 (Progress). If $\vdash p: \Delta$ and $\Delta ; \Delta_{p}^{r} ; \emptyset ; \emptyset \vdash e: C$, then either $e$ is a value or $e \longrightarrow p e^{\prime}$ for some $e^{\prime}$.

Proof. The proof is by induction on the typing rules.

(VAR-T) This case is empty since $\Pi=\emptyset$.

(PRE-OBJ-T) If the term is of the form $\mathcal{E}\{e\}$ with $e$ not a value, then it can be reduced by (CTX) and inductive hypothesis. Otherwise, the term is of the form $C(\bar{v})$, hence is a value.

(NEW-T) If the term is of the form $\mathcal{E}\{e\}$ with $e$ not a value, then it can be reduced by (CTX) and inductive hypothesis. Otherwise we have:

A: $\Delta ; \Delta^{r} ; \Gamma ; \Pi \vdash$ new $C\left(v_{1} \ldots v_{n}\right): C$

B: $\Delta ; \Delta^{r} ; \Gamma ; \Pi \vdash v_{i}: C_{i}^{\prime}$ for $i \in 1 . . n$

$\mathrm{C}: \Delta(C)=\left[\emptyset ; \Sigma^{o} ; C_{1} \ldots C_{n} ; C^{\prime}\right]$

D: $\Delta \vdash C_{i}^{\prime} \leq C_{i}$ for $i \in 1 . . n$

We can apply (OBJ-CREATION) since the implicit and explicit side conditions are verified:

- The term is of the form new $C(\bar{v})$ by $(\mathrm{A})$.

- $p(C)=[\emptyset|o|\{\tau \bar{\varphi} \mathrm{K}(\bar{x})\{\bar{e}\} \bar{\mu}\}]$ is verified by (C), Lemma 3.i, Lemma 3.ii.B, Lemma 3.ii.C. 
$-\bar{e}[\bar{v} / \bar{x}]$ is well-defined since

- \#( $\bar{v})=\#\left(\bar{C}^{\prime}\right)$ by $(\mathrm{B})$

- $\#(\bar{C})=\#\left(\bar{C}^{\prime}\right)$ by (D)

- $\#(\bar{x})=\#(\bar{C})$ by Lemma 3.ii.E

- $\#(\bar{v})=\#(\bar{x})$ by transitivity of equality

(CLIENT-FIELD-T) If the term is of the form $\mathcal{E}\{e\}$ with $e$ not a value, then it can be reduced by (CTX) and inductive hypothesis. Otherwise we have

A: $\Delta ; \Delta^{r} ; \Gamma ; \Pi \vdash v^{C_{0}} . F: C$

B: $\Delta ; \Delta^{r} ; \Gamma ; \Pi \vdash v^{C_{0}}: C_{0}$ (by (PRE-OBJ-T))

$\mathrm{C}: \operatorname{mtype}\left(\Delta, C_{0}, F\right)=C$

We can apply (CLIENT-FIELD) since the implicit and explicit side conditions are verified:

- The term is of the form $v^{C_{0}} \cdot F$ by (A).

- $p\left(C_{0}\right)=[\emptyset|o, F \mapsto f| \rho]$ by (B), (C), Lemma 5.(ii) and well-formedness of class $C_{0}$.

(CLIENT-INVK-T) If the term is of the form $\mathcal{E}\{e\}$ with $e$ not a value, then it can be reduced by (CTX) and inductive hypothesis. Otherwise we have

A: $\Delta ; \Delta^{r} ; \Gamma ; \Pi \vdash v^{C_{0}} \cdot M(\bar{e}): C$

B: $\Delta ; \Delta^{r} ; \Gamma ; \Pi \vdash v^{C_{0}}: C_{0}$

$\mathrm{C}: \operatorname{mtype}\left(\Delta, C_{0}, M\right)=\bar{C} \rightarrow C$

We can apply (CLIENT-INVK) since the implicit and explicit side conditions are verified:

- the term is of the form $v^{C_{0}} \cdot M(\bar{v})$ by $(\mathrm{A})$

$-p(C)=[\emptyset|o, M \mapsto m| \rho]$ by (B), (C), Lemma 5.ii

- $\left[\bar{\mu} ; v^{C_{0}} \mid m(\bar{v})\right]$ is well-formed since $m \in \rho$ by well-formedness of class $C_{0}$.

(BLOCK-T) we have:

A: $\Delta ; \Delta^{r} ; \emptyset ; \emptyset \vdash[\bar{\mu} ; v \mid e]: C$

B: $\Delta ; \emptyset ; \emptyset \vdash v: C^{\prime}$

C: $\Delta\left(C^{\prime}\right)=\left[\emptyset ; \Sigma^{o} ; \bar{C} ; C\right]$

D: $\Delta ; \Delta^{r} ; \Delta^{r}\left(C^{\prime}\right) ; \emptyset \vdash e: C$

The proof is divided in subcases, depending on the form of the inner $e$ :

- if the block contains a value, then it can be reduced by (EXT-BLOCK)

- else, if the block does not contains any sub-block:

- If $e$ is of the form $\mathcal{E}\{f\}$, then we have $\left[\bar{\mu} ; C\left(f_{1}=v_{1} \ldots f_{n}=v_{n}\right) \mid\right.$ $\mathcal{E}\{f\}] \longrightarrow p\left[\bar{\mu} ; C\left(f_{1}=v_{1} \ldots f_{n}=v_{n}\right) \mid v_{i}\right]$ by (INT-FIELD), since the implicit and explicit side conditions are verified:

$* H B(\mathcal{E})=\emptyset$ because there are no sub-blocks,

$* f=f_{i}$ by (D), Lemma 2.ii, Lemma 6.i.

- If $e$ is not of the form $e=\mathcal{E}\{f\}$, then, by Lemma 7:

$* e=\mathcal{E}\{x\}$ impossible because we are in a well-typed block

$* e=\mathcal{E}\left\{\left[\bar{\mu} ; v \mid e^{\prime}\right]\right\}$ impossible because there are no sub-blocks

$* e=\mathcal{E}\{m(\bar{v})\}:$ in this case, it must be $\left[\bar{\mu} ; v^{C} \mid \mathcal{E}\{m(\bar{v})\}\right] \longrightarrow p$ $\left[\bar{\mu} ; v^{C} \mid e[\bar{v} / \bar{x}]\left[v^{C} /\right.\right.$ this $\left.]\right]$

by (INT-INVK), since the implicit and explicit side conditions are verified: 
- $H B(\mathcal{E})=\emptyset$ because there are no sub-blocks.

- $\#(\bar{v})=\#(\bar{x})$ by Lemma 2. ii and Lemma 6 .ii

$* e=\mathcal{E}\left\{e^{\prime}\right\}$ and $e^{\prime}=v \cdot F$ or $e^{\prime}=v \cdot M(\bar{v})$ or $e^{\prime}=$ new $C((\bar{v}))$ :

in this case, names $\left(e^{\prime}\right)=\emptyset$, and we can apply (CTX), since:

$\Delta ; \Delta^{r} ; \Gamma ; \Pi \vdash e^{\prime}: C^{x}$ by Lemma 2. i; by Lemma 1 we have $\Delta ; \Delta^{r} ; \emptyset ; \emptyset \vdash$ $e^{\prime}: C^{x}$ since names $\left(e^{\prime}\right)=\emptyset$ and $e^{\prime}$ has no free variables (it is in a well-typed block). Now, $e^{\prime}$ is of a form which we have already proved can be reduced.

- if the block contains one or more sub-blocks: there exists an inner block that does not contain sub-blocks. Such sub-block is well-typed by Lemma 2 and it can be reduced by (CTX) because it falls in the previous case.

Theorem 6 (Subject reduction). If $\vdash p: \Delta, \Delta ; \Delta_{p}^{r} ; \Gamma ; \Pi \vdash e: C$, and $e \longrightarrow p$ $e^{\prime}$, then $\Delta ; \Delta_{p}^{r} ; \Pi ; e \vdash C^{\prime}$, and $\Delta \vdash C \leq C^{\prime}$.

Proof.

Note that $\vdash p: \Delta$ implies that for any class $C=C E$ (BASE-T) holds for $C E$.

The proof is by induction on the reduction rules. We show some cases.

(CLIENT-FIELD) By (CLIENT-FIELD) we know

$\mathrm{A}: v^{C_{0}} \cdot F \longrightarrow p\left[\bar{\mu} ; v^{C_{0}} \mid f\right]$

B: $p\left(C_{0}\right)=[\iota|o| \rho]$ with

$-\rho=\{\tau \bar{\varphi} \kappa \bar{\mu}\}$

C: $F: C^{\prime} \mapsto f \in O$

D: $C f \in \bar{\varphi}$ by well-formedness of $p\left(C_{0}\right)$

E: $\Delta \vdash C \leq C^{\prime}$ by $($ BASE-T)

It is typed by (CLIENT-FIELD-T) so

$\mathrm{F}: \Delta ; \Delta^{r} ; \Gamma ; \Pi \vdash v^{C_{0}} . F: C^{\prime}$

$\mathrm{G}: \Delta ; \Delta^{r} ; \Gamma ; \Pi \vdash v^{C_{0}}: C_{0}$

$\mathrm{H}: \operatorname{mtype}\left(\Delta, C_{0}, F\right)=C^{\prime}$

$\left[\bar{\mu} ; v^{C_{0}} \mid f\right]$ is typed by (BLOCK-T) (that uses (INT-FIELD-T)) because all the implicit and explicit side-conditions are verified:

$-\Delta ; \Delta^{r} ; \emptyset ; \emptyset \vdash v^{C_{0}}: C_{0}$ by $(\mathrm{G})$ and Lemma 1

$-\Delta ; \Delta^{r} ; \Delta^{r}\left(C_{0}\right) ; \emptyset \vdash f: C$ by (INT-FIELD-T), definition of $\Delta^{r}$ and (D).

$-\Delta ; \Gamma^{C}, \Gamma^{\bar{\mu}} ; C \vdash \bar{\mu}: \Gamma^{\bar{\mu}}$ by Lemma 2.ii.F and (BASIC-T)

We conclude that $\Delta ; \Delta^{r} ; \Gamma ; \Pi \vdash\left[\bar{\mu} ; v^{C_{0}} \mid f\right]: C$ and by (E) and (F) we prove the case.

(CLIENT-INVK) By (CLIENT-INVK) we know

A: $v^{C_{0}} \cdot M\left(v_{1} \ldots v_{n}\right) \longrightarrow p\left[\bar{\mu} ; v^{C_{0}} \mid m\left(v_{1} \ldots v_{n}\right)\right]$

B: $p\left(C_{0}\right)=[\iota|o| \rho]$ with

$-\rho=\{\tau \bar{\varphi} \kappa \bar{\mu}\}$

$\mathrm{C}: M: C_{1} \ldots C_{n} \rightarrow C \in O$

D: $C^{\prime} m\left(C_{1}^{\prime} x_{1}, \ldots, C_{n}^{\prime} x_{n}\right)\{$ return $e ;\} \in \bar{\mu}$ by well-formedness of $p\left(C_{0}\right)$

E: $\Delta \vdash C_{i} \leq C_{i}^{\prime}$ for $i \in 1 . . n$ and $\Delta \vdash C^{\prime} \leq C$

It is typed by (CLIENT-INVK-T) so

$\mathrm{F}: \Delta ; \Delta^{r} ; \Gamma ; \Pi \vdash v^{C_{0}}: C_{0}$.

G: $\Delta ; \Delta^{r} ; \Gamma ; \Pi \vdash v^{C_{0}} \cdot M\left(v_{1} \ldots v_{n}\right): C$. 
$\mathrm{H}: \Delta ; \Delta^{r} ; \Gamma ; \Pi \vdash v_{i}: C_{i}^{\prime \prime}$ for $i \in 1 . . n$.

I: $\operatorname{mtype}\left(\Delta, C_{0}, M\right)=C_{1} \ldots C_{n} \rightarrow C$.

$\mathrm{L}: \Delta \vdash C_{i}^{\prime \prime} \leq C_{i}$ for $i \in 1 . . n$.

$\mathrm{K}: \Delta \vdash C_{i}^{\prime \prime} \leq C_{i}^{\prime}$ for $i \in 1 . . n$ by $(\mathrm{L}),(\mathrm{E})$ and transitivity of subtyping relation.

$\left[\bar{\mu} ; v^{C_{0}} \mid m\left(v_{1} \ldots v_{n}\right)\right]$ is typed by (BLOCK-T) (that uses (INT-INVK-T)) because all the implicit and explicit side-conditions are verified:

$-\Delta ; \Delta^{r} ; \emptyset ; \emptyset \vdash v^{C_{0}}: C_{0}$ by (D) and Lemma 1.

$-\Delta ; \Delta^{r} ; \Delta^{r}\left(C_{0}\right) ; \emptyset \vdash m\left(v_{1} \ldots v_{n}\right): C$ by (INT-INVK-T), definition of $\Delta^{r},(\mathrm{D})$ and $(\mathrm{K})$.

$-\Delta ; \Gamma^{C}, \Gamma^{\bar{\mu}} ; C \vdash \bar{\mu}: \Gamma^{\bar{\mu}}$ by Lemma 2.ii.F and (BASIC-T)

We conclude that $\Delta ; \Delta^{r} ; \Gamma ; \Pi \vdash\left[\bar{\mu} ; v^{C_{0}} \mid m\left(v_{1} \ldots v_{n}\right)\right]: C^{\prime}$ and by (E) and (G) we prove the case.

Theorem 7. If $p \stackrel{\star}{\longrightarrow} p^{\prime}$, then $e \stackrel{\star}{\longrightarrow} p$ iff $e \stackrel{\star}{\longrightarrow} p^{\prime} v$.

To prove the theorem, we first of all define two congruence relations $\sim_{p}$ and $\sim$ on expressions, the former indexed on programs:

$-\sim_{p}$ is the least congruence relation s.t.

$\left[\hat{\iota}, n \mapsto \pi ; \bar{\mu} ; v^{C} \mid e\right] \sim_{p}\left[\hat{\iota}, \hat{\iota}^{\prime} ; \bar{\mu}\left[n^{\prime} / n\right], \bar{\mu}^{\prime} ; v^{C} \mid e\left[n^{\prime} / n\right]\right]$

if $\operatorname{lookup}_{p}\langle C, \pi\rangle=\left[\hat{\iota}^{\prime} ; \bar{\mu}^{\prime} \mid n^{\prime}\right]$.

$-\sim$ is the least congruence relation s.t.

$[\hat{\imath} ; \bar{\mu}, \mu ; v \mid e] \sim[\hat{\imath} ; \bar{\mu} ; v \mid e]$

if $\mu=C m(\overline{C x})\{$ return $e ;\}, m \notin$ names $(e) \cup$ names $(\bar{\mu})$.

The former congruence states that a block expression is equivalent to another where an association from internal name to path has been resolved by lookup, and path map and methods expanded. The expression on the left-hand-side, intuitively, is a lazy version which requires a further lookup of $\pi$ only when $n$ is needed, whereas in the right-hand-side this lookup has already been performed. The latter congruence states that a block expression is equivalent to another where a useless method has been removed.

Lemma 8. If $p \longrightarrow p^{\prime}, \operatorname{lookup}_{p}\langle N, \pi, C\rangle=\left[\hat{\imath} ; \bar{\mu}_{1} \mid n\right]$, then there exist $\left[\hat{\iota}^{\prime} ; \bar{\mu}_{1}^{\prime} \mid n\right]$, $\left[\emptyset ; \bar{\mu}_{2} \mid n\right],\left[\emptyset ; \bar{\mu}_{2}^{\prime} \mid n\right]$ s.t.

lookup $_{p^{\prime}}\langle N, \pi, C\rangle=\left[\hat{\iota}^{\prime} ; \bar{\mu}_{1}^{\prime} \mid n\right]$,

$\left[\hat{\imath} ; \bar{\mu}_{1} \mid n\right] \sim_{p}\left[\emptyset ; \bar{\mu}_{2} \mid n\right]$,

$\left[\hat{\iota}^{\prime} ; \bar{\mu}_{1}^{\prime} \mid n\right] \sim_{p^{\prime}}\left[\emptyset ; \bar{\mu}_{2}^{\prime} \mid n\right]$

$\left[\emptyset ; \bar{\mu}_{2} \mid n\right] \sim\left[\emptyset ; \bar{\mu}_{2}^{\prime} \mid n\right]$

Proof. By induction on the definition of $p \longrightarrow p^{\prime}$.

(CDEC1) We have

$C E \longrightarrow C E^{\prime}$,

$p \equiv p_{1}, C \mapsto C E \longrightarrow p^{\prime} \equiv p_{1}, C \mapsto C E^{\prime}$

We show, by induction on the definition of $C E \longrightarrow C E^{\prime}$, that, for any $N, \pi$ the statement of the lemma holds for the triple $\langle N, \pi, C\rangle$. This is enough to prove the thesis since other class names are not affected. 
(sum) We have

$$
\begin{aligned}
& C E=C E_{1}+C E_{2} \\
& C E_{1}=\left[\iota\left|o_{1}\right|\left\{\tau \bar{\varphi}_{1} \mathrm{~K}(\overline{C x})\left\{\overline{f_{1}=e_{1}}\right\} \bar{\mu}_{1}\right\}\right] \\
& C E_{2}=\left[\iota\left|o_{2}\right|\left\{\tau \overline{\varphi_{2}} \mathrm{~K}(\overline{C x})\left\{\overline{f_{2}=e_{2}}\right\} \bar{\mu}_{2}\right\}\right] \\
& C E^{\prime}=\left[\iota\left|o_{1}, o_{2}\right|\left\{\tau \bar{\varphi}_{1}, \bar{\varphi}_{2} \mathrm{~K}(\overline{C x})\left\{\overline{f_{1}=e_{1}}, \overline{f_{2}=e_{2}}\right\} \bar{\mu}_{1}, \bar{\mu}_{2}\right\}\right]
\end{aligned}
$$

Moreover, lookup $p\langle N, \pi, C E\rangle$ is defined only if $\left(o_{1}, o_{2}\right)(N)=n$ for some $n$. By well-formedness of $o_{1}, o_{2}$ this means that either $o_{1}(N)$ is defined or $o_{2}(N)$ is defined, but not both. Let us assume $o_{1}(N)=n$ (the other case is analogous). Then,

$$
\begin{aligned}
& \text { lookup }_{p}\langle N, \pi, C E\rangle=\left[\iota ; \alpha_{1}\left(\bar{\mu}_{1}\right) ; n \mid\right. \\
& ] \text { lookup }_{p}\langle N, \pi, C E\rangle=\left[\iota ; \alpha_{1}\left(\bar{\mu}_{1}\right), \alpha_{2}\left(\bar{\mu}_{2}\right) ; n \mid\right.
\end{aligned}
$$

and we get the thesis since, by well-formedness of $C E^{\prime}, n \in \operatorname{dom}\left(\iota, \alpha_{1}\left(\bar{\mu}_{1}\right)\right)$ and names $\left(\alpha_{1}\left(\bar{\mu}_{1}\right)\right) \cap \operatorname{dom}\left(\iota, \alpha_{2}\left(\bar{\mu}_{2}\right)\right)=\emptyset$.

(REDUCT) We have

$$
\begin{aligned}
& C E=\sigma^{\iota} \mid[\iota|o|\{\tau \bar{\varphi} \kappa \bar{\mu}\}]_{\mid \sigma^{o}} \\
& C E^{\prime}=\left[\sigma^{\iota} \circ \iota\left|o \circ \sigma^{o}\right|\{\tau \bar{\varphi} \kappa \bar{\mu}\}\right]
\end{aligned}
$$

Moreover, lookup $\langle\hat{N}, \pi, C E\rangle$ and lookup $p$ $\left\langle\hat{N}, \pi, C E^{\prime}\right\rangle$ are defined only if $\hat{N}=N$ and $o(N)=n$ for some $n$. Then, lookup $_{p}\langle N, \pi, C E\rangle=\left[\sigma^{\iota} \circ \iota ; \bar{\mu} ; n \mid\right.$

] lookup $_{p}\left\langle N, \pi, C E^{\prime}\right\rangle=\left[\sigma^{\iota} \circ \iota ; \bar{\mu} ; n \mid\right.$

]

and we get the thesis.

(FREEZE) We have

$C E=$ freeze $_{N}\left[\iota, n_{1}: T \mapsto N \ldots n_{k}: T \mapsto N|o|\{\tau \bar{\varphi} \kappa \bar{\mu}\}\right]$

$C E^{\prime}=\left[\iota|o|\left\{\tau \bar{\varphi} \kappa \bar{\mu}\left[o(N) / n_{1}\right] \ldots\left[n_{k} / o(N)\right]\right\}\right]$

$N \notin \operatorname{cod}(\iota)$

Moreover, lookup $_{p}\langle\hat{N}, \pi, C E\rangle$ and lookup $p\left\langle\hat{N}, \pi, C E^{\prime}\right\rangle$ are defined only if $\hat{N}=N^{\prime}$ and $o\left(N^{\prime}\right)=n^{\prime}$ for some $n^{\prime}$. Then, lookup $_{p}\left\langle N^{\prime}, \pi, C E\right\rangle=\left[\iota ; \bar{\mu}, n_{i} \stackrel{i \in I}{\mapsto} \pi ; n^{\prime} \mid\right.$

]$_{\text {lookup }}\left\langle N^{\prime}, \pi, C E^{\prime}\right\rangle=\left[\iota ; \bar{\mu}, n_{i} \stackrel{i \in I}{\mapsto} \bar{\mu}\left(n^{\prime}\right) ; n^{\prime} \mid\right.$ ]

Since $C E$ is the $\pi$-subterm of $p(C), \operatorname{lookup}_{p}\langle\pi, \Lambda, C\rangle=\operatorname{lookup}_{p}\langle\pi, \pi, C E\rangle=$ $\left[\iota ; \bar{\mu}, n_{i} \stackrel{i \in I}{\mapsto} \pi ; n \rrbracket\right.$ with $n=o(N)$, hence the thesis follows.

(WRAPPING) Trivial.

(CTX) The proof is by structural induction on the context. We show the following case (the others are analogous): $\begin{aligned} & C E_{1} \longrightarrow C E_{1}^{\prime} \\ & C E_{1}+C E_{2} \longrightarrow C E_{1}^{\prime}+C E_{2}\end{aligned}$

We have to prove that lookup $p$ $\left\langle\hat{N}, \pi, C E_{1}+C E_{2}\right\rangle \sim_{p, C} \operatorname{lookup}_{p}\left\langle\hat{N}, \pi, C E_{1}^{\prime}+C E_{2}\right\rangle$ for all $\hat{N}$ and $\pi$. There are two cases.

- lookup $p\left\langle\hat{N}, \pi, C E_{1}+C E_{2}\right\rangle=$ lookup $_{p}\left\langle\hat{N}, \pi .1, C E_{1}\right\rangle$. In this case, by inductive hypothesis

- $\operatorname{lookup}_{p}\left\langle\hat{N}, \pi, C E_{1}+C E_{2}\right\rangle=\operatorname{lookup}_{p}\left\langle\hat{N}, \pi .1, C E_{2}\right\rangle$.

(CDEC2) If $p \longrightarrow p^{\prime}$, lookup $_{p}\langle N, C\rangle$ is defined, then lookup $p$ $\langle N, C\rangle \sim_{p}$ lookup $_{p^{\prime}}\langle N, C\rangle$. 
1. This can be proved by induction on the definition of $C E \longrightarrow C E^{\prime}$.

2. By induction on the definition of $p \longrightarrow p^{\prime}$.

Then, Theorem 4 follows as a corollary of the following.

Theorem 8. If $p \longrightarrow p^{\prime}, e_{1} \longrightarrow p e_{1}^{\prime}$, and $e_{1} \sim e_{2}$, then there exists $e_{2}^{\prime}, e_{1}^{\prime \prime}, e_{2}^{\prime \prime}$ s.t.:

$$
\begin{array}{ccc}
e_{1} \longrightarrow p & e_{1}^{\prime} \sim_{p} & e_{1}^{\prime \prime} \\
2 & \\
e_{2} \longrightarrow p^{\prime} & e_{2}^{\prime} \sim_{p^{\prime}} & e_{2}^{\prime \prime}
\end{array}
$$

Proof. 